\title{
Effects of the Spin-Orbit and Tensor Interactions on the $M 1$ and E2 Excitations in Light Nuclei
}

\author{
M. S. Fayache ${ }^{1,3}$, Y.Y. Sharon ${ }^{1}$ and L. Zamick ${ }^{1,2}$, P. von Neumann-Cosel ${ }^{2}$ and A. Richter ${ }^{2}$ \\ (1) Department of Physics and Astronomy, Rutgers University, Piscataway, New Jersey 08855 \\ (2) Institut fur Kernphysik, Technische Hochschule, Darmstadt, D-64289 Darmstadt, Germany \\ (3) Département de Physique, Faculté des Sciences de Tunis, Tunis 1060, Tunisia
}

(July 2, 2021)

The effects of varying the spin-orbit and tensor components of a realistic interaction on $M 1$ excitation rates and $B(E 2)^{\prime} s$ are studied on nuclei in the $0 p$ and $1 s-0 d$ shells. Not only the total $M 1$ but also the spin and orbital parts separately are studied. The single-particle energies are first calculated with the same interaction that is used between the valence nucleons. Later this stringent condition is relaxed somewhat and the $1 s$ level is raised relative to $0 d$. For nuclei up to ${ }^{28} \mathrm{Si}$, much better results i.e stronger $B(M 1)$ rates are obtained by increasing the strength of the spin-orbit interaction relative to the free value. This is probably also true for ${ }^{32} S$, but ${ }^{36} A r$ presents some difficulties. The effects of weakening the tensor interaction are also studied. On a more subtle level, the optimum spin-orbit interaction in the lower half of the $s-d$ shell, as far as $M 1$ excitations are concerned, is substantially larger than the difference $E\left(J=3 / 2^{+}\right)_{1}-E\left(J=5 / 2^{+}\right)_{1}=5.2 \mathrm{MeV}$ in ${ }^{17} \mathrm{O}$. A larger spin-orbit splitting is also needed to destroy the triaxiality in ${ }^{22} N e$. Also studied are how much $M 1$ orbital and spin strength lies in an observable region and how much is buried in the grass at higher energies. It is noted that for many nuclei the sum $B(M 1)_{\text {orbital }}+B(M 1)_{\text {spin }}$ is very close to $B(M 1)_{\text {total }}$, indicating that the summed cross terms are very small.

\section{INTRODUCTION}

Our purpose is to see how various components of the realistic nucleon-nucleon interaction affect nuclear properties. In particular, we shall investigate magnetic dipole excitations to $J=1^{+}$states of even-even nuclei -these are spin and orbital modes, and as a counter point we shall look at low lying $2^{+}$states which for deformed open shell nuclei have very little spin content. We will examine selected nuclei in the $0 p$ and $1 s-0 d$ shell.

At first thought it may seem that such an investigation is unnecessary because excellent fits have been obtained by Cohen and Kurath [1] in the $p$ shell and by Wildenthal and Brown [2] in the $1 s-0 d$ shell. These works are of extreme importance if for no other reason than that they showed that the shell model works well beyond many peoples expectations -well enough in fact so that the authors in collaboration with the experimental groups could meaningfully go one step further and discuss effects of exchange currents on $M 1$ 's in a systematic way [3, 4 . The works brought order out of the myriad of energy levels that were discussed by experimentalists.

However, the truly excellent fits were obtained with empirical effective interactions e.g. the Wildenthal interaction in the $s d$ shell [5]. Such an interaction implicitly has in it several effects e.g. core polarization, relativistic phenomenology etc. It is impossible to sort out these effects from an empirical interaction expressed as hundreds of matrix elements.

Our intention is to start with a realistic interaction which will admittedly not give as good a fit to the data as the above mentioned empirical interactions. But the discrepancies will show what is missing and what has to be done. The interaction that we use -the $(x, y)$ interaction- has been discussed elsewhere [6]. Suffice it to say that it is written as follows:

$$
V(r)=V_{c}(r)+x V_{\text {s.o. }}+y V_{t}
$$


where s.o. stands for the two-body spin-orbit interaction and $t$ for the tensor interaction and $V_{c}(r)$ is everything else especially the central interaction. For $x=1, y=1$, the interaction gives a good fit to the Bonn A matrix elements [6]. We can study the effects of changing the spin-orbit and tensor interactions by varying $x$ and $y$.

Most calculations which claim to use realistic interactions are really doing hybrid calculations in which the residual two-body matrix elements are carefully calculated but the single-particle energies are obtained from experiment. Here on the other hand we shall use the same interaction to calculate the single-particle matrix elements as was used to calculate the residual interaction. We feel this is the only way to really find out the effects of such an interaction. However, to overcome the deficiencies of using harmonic oscillator wavefunctions, we will also consider one case in which the relative spacing of the $1 s$ and $0 d$ single-particle energies are changed (likewise for $p$ and $0 f$ ).

We shall perform calculations with four sets of $(x, y):(1,1),(1.5,1),(1 ., 0.5)$ and $(1.5,0.5)$. We are motivated in these choices by the ideas presented by many that the spin-orbit interaction inside a nucleus should be stronger than what is deduced form nucleon-nucleon scattering and the tensor interaction weaker. We shall also consider one more case in which, for the $1 s-0 d$ shell, the $1 s$ single-particle energy is moved up by $2 \mathrm{MeV}$ relative to the $0 d$ centroid $\left(\Delta \epsilon_{s}=2 \mathrm{MeV}\right)$. This is to overcome the defects of using harmonic oscillator wavefunctions, and is equivalent to the Nilsson prescription of adding a $D \vec{l} \cdot \vec{l}$ term to the Hamiltonian.

A stronger spin-orbit interaction arises for example in the Walecka model, also known as Dirac phenomenology [7. There is a parameter in the theory called the Dirac effective mass which arises from sigma exchange. The ratio $m_{D} / m$ is less than one and the spin-orbit interaction is inversely proportional to $m_{D} / m$. In another formulation of Wiring et. al., the stronger spin-orbit interaction comes from a three-body interaction 8].

Concerning the weakening of the tensor interaction inside a nucleus, two basic ideas have been advanced. One is the universal scaling argument by G.E. Brown and collaborators [9]. The basic idea is that all mesons inside the nucleus except for the pion become less massive. Hence the range of the nucleon-nucleon interaction due to a given boson exchange becomes larger. In particular, the $\rho$ meson exchange will be of larger range. This gives a larger repulsive contribution to the tensor interaction which will cancel the attraction due to the pion. The increased cancellation will result in a weaker tensor interaction.

On the other hand Fayache, Zheng and Zamick have advocated a self-weakening mechanism for the tensor interaction [10]. They show that the effects of higher-shell admixtures makes the tensor interaction in a $\Delta N=0$ (one major shell) space appear weaker. Thus, at least for nuclear structure calculations, no mechanism beyond higher shell admixtures seems to be needed.

However, the theory of Brown et. al. [9] also pertains to nucleon-nucleus scattering problems at intermediate to high energies. Here the anomalies for the $q^{2}$ behaviour of several Wolfenstein parameters can be explained by the universal scaling arguments, but it has not been demonstrated that better nuclear structure calculations would also resolve these anomalies.

\section{THE MAGNETIC DIPOLE OPERATOR}

In what follows we discuss present calculations for what we call $B(M 1)_{\text {physical }}, B(M 1)_{\text {orbital }}$ and $B(M 1)_{\text {spin }}$. In terms of a magnetic dipole operator, the $B(M 1)$ is defined as

$$
\frac{1}{\left(2 J_{i}+1\right)} \sum_{M_{f}, \mu, M_{i}}\left|\left\langle\Psi_{M_{f}}^{J_{f}} A_{\mu} \Psi_{M_{i}}^{J_{i}}\right\rangle\right|^{2}
$$

where

$$
\vec{A}=\sqrt{3 / 4 \pi}\left[\sum_{\text {protons }}\left(g_{l \pi} \overrightarrow{l_{i}}+g_{s \pi} \overrightarrow{s_{i}}\right)+\sum_{\text {neutrons }}\left(g_{l \nu} \overrightarrow{l_{i}}+g_{s \nu} \overrightarrow{s_{i}}\right)\right]
$$


For the three cases considered, the following parameters were used:

$\begin{array}{lllll}\text { Physical } & g_{l \pi}=1 & g_{s \pi}=5.586 & g_{l \nu}=0 & g_{s \nu}=-3.826 \\ \text { Orbital } & g_{l \pi}=1 & g_{s \pi}=0 & g_{l \nu}=0 & g_{s \nu}=0 \\ \text { Spin } & g_{l \pi}=0 & g_{s \pi}=5.586 & g_{l \nu}=0 & g_{s \nu}=-3.826\end{array}$

In other words, for the $B(M 1)_{\text {orbital }}$ we set the spin $g$ factors to zero and for $B(M 1)_{\text {spin }}$ we set the orbital $g$ factors to zero.

One can also write the magnetic moment operator as $\vec{A}=\vec{A}_{\text {scalar }}+\vec{A}_{\text {vector }} \tau_{z}$ where $\tau_{z}=+1$ for the proton and -1 for the neutron. For the physical operator,

$$
g_{s}(\text { scalar })=\frac{g_{s \pi}+g_{s \nu}}{2}=0.88
$$

and

$$
g_{s}(\text { vector })=\frac{g_{s \pi}-g_{s \nu}}{2}=4.706
$$

Both $g_{l}$ (scalar) and $g_{l}$ (vector) are equal to 0.5 . Because $g_{s}$ (vector) is much larger than $g_{s}$ (scalar), $T=0$ to $T=1$ transitions in $N=Z$ nuclei tend to be much larger that $T=0 \rightarrow T=0$ transitions. The ratio $\left(\frac{g_{s}(v e c t o r)}{g_{s}(\text { scalar })}\right)^{2}$ is equal to 81 .

There are some interesting limiting cases for $M 1$ matrix elements. For example, in the $S U(4)$ limit, all spin matrix transition matrix elements from the $J=0^{+}$ground state (both isoscalar and isovector) will vanish. The isoscalar orbital transition will also vanish. All that is left is the isovector orbital excitations known as scissors modes.

For strongly deformed nuclei one approaches this limit. The nuclei in the lower part of the $s-d$ shell e.g. ${ }^{20} N e$ and ${ }^{24} \mathrm{Mg}$ are strongly deformed and so we should look for such selection rules there. However, the spin contributions will not be negligible because $\frac{g_{s}(\text { vector })}{g_{l}(\text { vector })}=9.41$, and even if the spin matrix elements are strongly suppressed they start out with a big advantage.

\section{THE SINGLE-PARTICLE ENERGIES}

As mentioned in the introduction, we do not perform a hybrid calculation. We first obtain the single-particle energies with the same interaction that is used for the two-particle matrix elements. More to the point, in our shell model, we perform a calculation for say ${ }^{20} \mathrm{Ne}$ not merely as four nucleons in the $1 s-0 d$ shell but rather as twenty nucleons with sixteen in the closed $0 s$ and $0 p$ shells. This allows for valence-core interactions which implicitly generate the single-particle energies. (In a later section we relax this stringent condition by moving the $1 s$ single-particle level relative to $0 d$ in order to overcome the defects of using harmonic oscillator wavefunctions).

In Table I we present the single-particle energies obtained for the $(x, y)$ interaction with $x=1, y=1$ in both a small space and a large space. By the former we mean that the ${ }^{16} \mathrm{O}$ core is inert. In the large space, we allow $2 \hbar \omega$ excitations in the shell model diagonalization and we set the lowest state to zero [11].

We see one defect in that the lower $l$ states come down too low relative to the higher $l$ states. Whereas in the calculation the $1 s_{1 / 2}$ comes below $0 d_{5 / 2}$, for ${ }^{17} O$ we know it's the other way around. Likewise the $1 p_{3 / 2}$ comes 0.94 $\mathrm{MeV}$ below $0 f_{7 / 2}$ whereas experimentally the $1 p_{3 / 2}$ is about $2 \mathrm{MeV}$ above $0 f_{7 / 2}$. This tendency of the low $l$ to come too low has the effect of making nuclei more deformed than they are experimentally.

The calculated spin-orbit splitting for ${ }^{15} \mathrm{O}$ in the small space is $5.07 \mathrm{MeV}$, somewhat smaller than the splitting between the $J=3 / 2^{-}$excited state of ${ }^{15} \mathrm{O}$ and the $J=1 / 2^{-}$ground state. The latter is $6.1 \mathrm{MeV}$. For ${ }^{17} \mathrm{O}$ the 
splitting $J=3 / 2^{-}-J=1 / 2^{-}$is $5.562 \mathrm{MeV}$ in the calculation, which compares well with the empirical splitting of $5.2 \mathrm{MeV}$.

Going to the large space does not increase the splitting significantly. For $A=15$ the value is $5.679 \mathrm{MeV}$ while for $A=17$ it is $5.662 \mathrm{MeV}$.

An interesting question to ask is whether the empirical procedure above of identifying the spin-orbit splitting in say ${ }^{17} O$ with $E\left(J=3 / 2^{-}\right)-E\left(J=5 / 2^{-}\right)$is valid. We shall discuss this in the context of the systematics of the magnetic dipole transitions that we calculate.

\section{RESULTS FOR $M 1$ AND $E 2$ EXCITATION RATES AS A FUNCTION OF $X$ AND $Y$}

\section{(a) The Effects of the Spin-Orbit and Tensor Interactions on the Spin $B(M 1)$}

For all nuclei considered, the effect of increasing the spin-orbit strength parameter $x$ from 1 to 1.5 causes the spin $B(M 1)$ (also denoted by $B_{\sigma}$-last column of Table II) to increase. In ${ }^{12} C$ the increase (for $y=1$ ) is from 0.91 to 2.07;

in ${ }^{20} \mathrm{Ne}$ from 0.59 to 1.28 ; in ${ }^{24} \mathrm{Mg}$ from 1.73 to 3.35 , in ${ }^{32} \mathrm{~S}$ from 2.72 to 8.52 ; and in ${ }^{36} \mathrm{Ar}$ from 2.25 to 5.20 . The change is especially large in ${ }^{32} S$.

The increase in $B_{\sigma}$ can be understood by noting that this quantity increases as we go from the $L S$ limit to the $j-j$ limit. Indeed, in the $S U(4)$ limit, the spin $B(M 1)$ will vanish.

In all cases considered, decreasing the tensor interaction also causes the spin $B(M 1)$ to increase. This is consistent with the old observation of C.W. Wong [12] that the tensor interaction in an open shell nucleus acts to some extent like a spin-orbit interaction of the opposite sign as the main spin-orbit term. Thus, in ${ }^{24} \mathrm{Mg}$, when we go from $x=1 y=1$ to $x=1 y=0.5$ the $B(M 1)_{\text {spin }}$ (in units of $\mu_{N}^{2}$ ) goes from 1.73 to 1.94 ; and in going from $x=1.5 y=1$ to $x=1.5 y=0.5$, we find that $B(M 1)_{\text {spin }}$ increases from 3.35 to 4.04 . There is a similar behaviour for all other nuclei considered. The effect of varying the tensor strength $y$ is not quite as dramatic as that of varying the spin-orbit strength $x$.

It should be emphasized that there are basic differences between the tensor and the spin-orbit interactions. For a closed $L S$ plus or minus one nucleon, the tensor interaction gives no contribution to the single-particle energy in first order whilst the two-body spin orbit interaction does. The latter gives the majority amount of the spin-orbit splitting e.g. of $d_{3 / 2}$ and $d_{5 / 2}$ in ${ }^{17} \mathrm{O}$. What we are discussing in the above paragraph is an open shell effect.

To summarize, for the cases considered we get the smallest value of $B(M 1)_{\text {spin }}$ (and also $B(M 1)_{\text {physical }}$ ) for $x=1 y=1$ and the largest value for $x=1.5 y=0.5$ i.e. a stronger spin-orbit and a weaker tensor interaction than what is furnished by large $G$ matrix elements.

(b) The Effects of the Spin-Orbit and Tensor Interactions on the Orbital $B(M 1)$

The effect of varying $x$ and $y$ on the orbital summed strength is more complicated than for the spin $B(M 1)$. The effects are however not insignificant.

For $N=Z$ nuclei, we find that the effects become more important as we go the end of the shell. For ${ }^{20} \mathrm{Ne}$, the effect of changing $x$ and $y$ are very small. For the four sets of $x$ and $y$, the values of $B_{l}$ are $0.95,1.09,0.96$, and 1.10 (in the order as shown in Table II). For ${ }^{32} S$, the results are 2.32, 1.57, 2.17 and 1.15; and for ${ }^{36} A r$ they are 1.44, 0.95, 1.33 and 0.87. We also see that increasing the spin-orbit strength for ${ }^{24} \mathrm{Mg},{ }^{32} S$ and ${ }^{36} \mathrm{Ar}$ decreases the summed orbital strength. Decreasing $y$ (the tensor strength) in some cases (e.g. ${ }^{20} \mathrm{Ne}$ ) slightly increases the orbital strength, but in most cases, it causes a decrease in this strength. For example in ${ }^{32} S$, going from $x=1 y=1$ to $x=1 y=0.5$ the orbital strength decreases slightly from 2.32 to 2.17 ; whilst from $x=1.5 y=1$ to $x=1.5 y=0.5$ the orbital strength decreases from 1.57 to $1.15 \mu_{N}^{2}$.

(c) The Physical $B(M 1)$-additional observations 
The dependence of the physical $B(M 1)$ on $x$ and $y$ is qualitatively similar to that of $B(M 1)_{s p i n}$. In general, increasing $x$ and decreasing $y$ will cause the summed strength $B(M 1)_{\text {physical }}$ to increase.

Recalling that $\frac{g_{s} \text { (isovector) }}{g_{l} \text { (isovector) }}=9.41$, this fact alone would cause $B(M 1)_{\text {spin }}$ to be about 85 times larger than $B(M 1)_{\text {orbital }}$. However, overall in Table II, $B(M 1)_{\text {orbital }}$ and $B(M 1)_{\text {spin }}$ are comparable in magnitude. For example, for $x=1 y=1$ in ${ }^{24} M g, B(M 1)_{\text {orbital }}=2.08$ and $B(M 1)_{\text {spin }}=1.73$. It is true that when we change to $x=1.5 y=0.5$ the $B(M 1)_{\text {spin }}$ becomes larger than $B(M 1)_{\text {orbital }}$ (4.04 vs. 1.69), but the ratio is much less than 85 .

These and other numbers in Table II confirm what was said in a previous section -that the spin matrix elements are strongly suppressed because in deformed nuclei one moves away from the $j j$ limit towards the $L S S U(4)$ limit.

(d) The Relationship of the Physical $B(M 1)$ to the Sum of the Orbital $B(M 1)$ and the Spin $B(M 1)$

Let us consider the $B(M 1)$ transition from the ground state $0^{+}$to any single $1^{+}$state. Then the physical $B(M 1)$ will be the sum of the orbital $B(M 1)$ and the spin $B(M 1)$ plus their cross terms which in principle may be positive or negative. In Table III the spin, orbital and physical $B(M 1)$ results each represent the sum (over all the $1^{+}$states in our space) of the corresponding single-state $B(M 1)$ 's. Let us now treat the ${ }^{22} N e$ results as one unit, summing the $T=1 \rightarrow T=1$ (both isovector and isoscalar) and $T=1 \rightarrow T=2$ results. Then for all the nuclei that we consider and for all the $x, y$ combinations (all 28 cases except for the $x=1 y=1$ case in ${ }^{44} \mathrm{~T} i$ with a difference of less than $1 \%$ ), the physical $B(M 1)$ is less than the sum of the spin $B(M 1)$ and orbital $B(M 1)$. Remarkably, in all the ${ }^{20} N e$, ${ }^{22} \mathrm{Ne}$ and ${ }^{44} \mathrm{Ti}$ cases the difference is less than $3 \%$; for ${ }^{24} \mathrm{Mg}$ less than $6 \%$; all these cases are in the first half of the major shell. For the other three cases ${ }^{12} C,{ }^{32} S$ and ${ }^{36} \mathrm{Ar}$ (all in the second half of major shells), the difference is still always less than $20 \%$, with the physical $B(M 1)$ being less than the sum of the other two. Just to illustrate, in ${ }^{20} N e$ the four respective spin $B(M 1)$ plus orbital $B(M 1)$ sums (i.e. for the four $x, y$ combinations) are $1.54,2.37,1.59$ and 2.59 ; the corresponding physical $B(M 1)$ values are $1.53,2.34,1.57$ and 2.54 !

\section{(e) Summed Strengths for Magnetic Dipole Excitations}

In Tables III and IV we compare the summed strength (somewhat arbitrarily) to the first ten states, and we also give the total sum. We limit ourselves to the case $x=1.5, y=0.5$. In Table IV we also have $x=1.5 y=0.5$ but we have $\Delta \epsilon_{s}=2 \mathrm{MeV}$.

Concerning the orbital strength we find in some cases a significant difference between the summed strength to all states and the sum to the first ten states. As seen in Table III for ${ }^{20} \mathrm{Ne}$ the difference is not so large (1.10 vs. 0.90), for ${ }^{22} \mathrm{Ne} T=1 \rightarrow T=1$ there is a factor of two difference (0.46 vs. 0.23). For ${ }^{24} \mathrm{Mg}$ and ${ }^{32} S$ the differences are also large (2.02 vs. 1.39 and 1.15 vs. 0.53 respectively).

This is of relevance to works on relations between summed orbital (scissors mode) strength and $B(E 2)_{0_{1} \rightarrow 2_{1}^{+}}$in deformed nuclei. What is precisely meant by the summed $M 1$ orbital strength? Experimentally it is very difficult if not impossible to pick up the orbital "grass" which presumably sets in at the very least by the time we have come to the first ten states if not earlier. What then do the sum rules mean if experimentally not all orbital strength is included?

\section{(f) Comprehensive List of $M 1$ Rates}

In Tables V and VI we give a comprehensive list of calculated values of excitation energy $(\mathrm{MeV}), B_{l}, B_{\sigma}$ and $B(M 1)$ in units of $\mu_{N}^{2}$ for the four sets of $(x, y)-(1,1),(1.5,1),(1,0.5)$ and $(1.5,0.5)$. In order not to drown ourselves in details, we limit Table $\mathrm{V}$ to states for which $B(M 1)$ is greater or equal to $0.3 \mu_{N}^{2}$ (there is one exception in ${ }^{20} \mathrm{Ne}$ $x=1, y=0.5$ where the strengths to two nearly degenerate states is given). However, there are other states of interest which are put into Table VI. For example, there are states with substantial orbital strengths and spin strengths but the destructive interference of the orbital and spin amplitudes causes $B(M 1)$ to be very small. In the case of ${ }^{36} A r$, such an 'invisible' state is lower in energy than the lowest strongly excited state.

On the whole, the behaviour in Table $\mathrm{V}$ is similar to that of Table II where the total summed strength is given. That is to say, in going from $(x, y)=(1,1)$ to $(1.5,1)$ the values of $B_{\sigma}$ and $B(M 1)$ increase. In making the analysis 
however, we have to take note that the fragmentation can be different for different values of $(x, y)$.

The fragmentation mentioned above is greater in some nuclei than in others. Whereas for ${ }^{8} \mathrm{Be}$ and ${ }^{12} \mathrm{C}$ there is only one state with a calculated value of $B(M 1)$ greater than $0.3 \mu_{N}^{2}$, in ${ }^{32} S$ and in ${ }^{36} A r$ there are four (with $x=1.5, y=0.5)$. In ${ }^{32} S$ states calculated to be at 10.05, 11.21, 13.01 and $13.81 \mathrm{MeV}$ have values of $B(M 1) 2.91$, $5.34,0.68$ and $0.44 \mu_{N}^{2}$ respectively. In ${ }^{36} \mathrm{Ar}$ states at $11.91,12.97,13.73$ and $17.65 \mathrm{MeV}$ have $B(M 1)$ values of 2.62 , $1.46,0.55$ and $0.49 \mu_{N}^{2}$. For ${ }^{36} A r$ the orbital strengths to these states is very small -it is mainly spin strength.

Table VI shows states with very weak $B(M 1)$ 's but for which $B_{l}$ and/or $B_{\sigma}$ are fairly large. The destructive interference between the orbital and spin amplitudes causes $B(M 1)$ to be very small. In the following discussion, we will be referring to calculated results for $x=1.5, y=0.5$ (not experiment).

In some cases it is the lowest $1^{+} T=1$ state for which this destructive interference occurs. This is especially true for ${ }^{36} \mathrm{Ar}$ where the lowest calculated $J=1^{+} T=1$ state for $x=1.5 y=0.5$ is at $7.80 \mathrm{MeV}$ with $B(M 1)$ negligibly small $\left(<5 \times 10^{-3} \mu_{N}^{2}\right)$. The next $1^{+} T=1$ state is over $4 \mathrm{MeV}$ higher in energy. This state is at $11.91 \mathrm{MeV}$ and has $B(M 1)=2.62 \mu_{N}^{2}$. The $7.80 \mathrm{MeV}$ state has significant $B_{l}$ and $B_{\sigma}$ values: $0.305 \mu_{N}^{2}$ and $0.320 \mu_{N}^{2}$ respectively. The amplitudes are nearly equal but of opposite sign, thus causing $B(M 1)$ to nearly vanish.

In ${ }^{8} \mathrm{Be}$ and ${ }^{20} \mathrm{Ne}$, the lowest states have the largest $B(M 1)$ values, but not too high above these are the 'invisible' states with substantial $B_{l}$ and $B_{\sigma}$ but negligible $B(M 1)$. Thus from a pure $\left(e, e^{\prime}\right)$ experiment one will miss considerable orbital strength which is buried in these invisible states. Only with a combination of $\left(e, e^{\prime}\right)$ and another probe e.g. $\left(p, p^{\prime}\right)$ can one unravel the spin and orbital content of the $M 1$ strength.

(g) $B(E 2)$ Rates

In Table VII we present results for summed isoscalar and isovector $B(E 2)$ for the four different sets of $(x, y)$. The general $E 2$ operator is

$$
\sum_{\pi} e_{p} r^{2} Y_{2, \mu}+\sum_{\nu} e_{n} r^{2} Y_{2, \mu}
$$

For the isoscalar case we take $e_{p}=1.5, e_{n}=0.5$ and for the isovector case $e_{p}=1, e_{n}=-1$.

It should be noted that, for transitions $J=0^{+} T=0 \rightarrow J=2^{+} T=1$, the overwhelming amount of the strength goes to the lowest $J=2^{+}$state. For example, for ${ }^{20} N e$ (using $x=1, y=1$ ) the value of $B(E 2) \uparrow$ to the lowest $2^{+}$ state at $2.92 \mathrm{MeV}$ is $299.4 \mathrm{e}^{2} \mathrm{fm}^{4}$ whilst the total sum is $315.1 \mathrm{e}^{2} \mathrm{fm}^{4}$. This seems to be true for all nuclei except ${ }^{22} \mathrm{Ne}$ where there is considerable fragmentation between the first two $2^{+}$states. For $x=1, y=1$, the value of $B(E 2)$ is $198.9 \mathrm{e}^{2} \mathrm{fm}^{4}$ to the $2_{1}^{+}$state at $2.27 \mathrm{MeV}$ and $113.4 \mathrm{e}^{2} \mathrm{fm}^{4}$ to the $3.10 \mathrm{MeV}$ state. The sum of these two strengths $\left(312.3 \mathrm{e}^{2} \mathrm{fm}^{4}\right)$ is however close to the total strength $\left(352.7 \mathrm{e}^{2} \mathrm{fm}^{4}\right)$.

It should be noted that for most nuclei considered here, the sensitivity of $B(E 2)$ to $x$ and $y$ is much less than it is for isovector $B(M 1)^{\prime} s$. For example, for ${ }^{20} \mathrm{Ne}$ the four values of $B(E 2)_{\text {isoscalar }}$ are $315.1,313.5,316.0$ and 311.1 $e^{2} \mathrm{fm}^{4}$ respectively.

This can be understood from the fact that the strong central interaction causes the low lying $2^{+}$states to be dominantly $S=0$ states. The expectation values of the spin-orbit and tensor interactions for pure $S=0$ states are zero.

For the ${ }^{32} S$ nucleus however, there is considerable sensitivity of the $B(E 2)_{\text {isoscalar }}$ to $x$ and $y$. For this nucleus there must be a lot of the $S=1$ component in the ground state.

\section{EFFECTS OF RAISING THE $1 S$ LEVEL WITH RESPECT TO THE $0 D$ LEVEL}

In our calculations for nuclei in the $1 s-0 d$ shell the calculated physical $B(M 1)$ values, especially with the $x=1 y=1$ interaction, were often smaller than the corresponding measured experimental values. This suggested to us that for these nuclei we should further investigate one additional aspect of our calculations, namely the energy separation of 
the single-particle $1 s$ and $0 d$ levels. From Table I it appears that the $1 s$ level is too low in energy relative to the centroid of the $d_{5 / 2}$ and $d_{3 / 2}$ levels. The fault may lie with the fact that we are using harmonic oscillator wavefunctions to evaluate matrix elements of the two-body interaction.

To study the above effect we redid our calculations with the $x=1.5 y=0.5$ interaction for the $1 s-0 d$ nuclei with the $1 \mathrm{~s}$ level raised by an additional $2 \mathrm{MeV}$ above the $0 d$ level. This correction is equivalent to adding a term $D \vec{l} \cdot \vec{l}$ to the single-particle spectrum, as was done in the Nilsson model. To calculate the best value of the energy difference to adopt, we would have proceeded as follows. Our calculations for the single-particle energies in ${ }^{17} O$ using the $x=1 y=1$ interaction (see Table I) yielded the following energies (in $M e V$ ): $\epsilon_{s_{1} / 2}=0, \epsilon_{0 d_{5} / 2}=0.119$, $\epsilon_{0 d_{3} / 2}=5.681$. These place the centroid of the $0 d$ levels at $2.34 \mathrm{MeV}$ above the $1 \mathrm{~s}$. Experimentally, we find the single-particle energies (in $\mathrm{MeV}$ ) in ${ }^{17} \mathrm{O}$ to be $\epsilon_{d_{5} / 2}=0, \epsilon_{1 s_{1} / 2}=0.871$, and $\epsilon_{d_{3} / 2}=5.09$. This places the $0 d$ centroid at $2.03 \mathrm{MeV}$, or $1.16 \mathrm{MeV}$ above the $1 \mathrm{~s}$. Accordingly, the $1 \mathrm{~s}$ should have been raised by $(2.35-1.16)=1.19 \mathrm{MeV}$. We chose however chose $2 \mathrm{MeV}$ rather than $1.19 \mathrm{MeV}$.

In Table IV we present the effects of raising the $1 s$ level relative to the $0 d$ level for the $x=1.5 y=0.5$ case. In this table we consider the summed $B(M 1)$ values (physical, orbital and spin) for transitions from the ground state to the lowest $500 J=1^{+} T=1$ states (or a smaller number if fewer states exist in our space). From the table, we see that the net effect of raising the $1 s_{1 / 2}$ level is larger in the first half of the shell. Perhaps this is because in the second half of the shell, the $1 s_{1 / 2}$ level plays a more minor role in excitations, being nearly fully occupied in both the ground state and the excited $J=1^{+} T=0$ states. In the first half of the shell, the total summed spin $M 1$ strength $\left(B_{\sigma}\right)$ increases by about $30 \%$ in three cases: ${ }^{20} N e,{ }^{22} N e(T=1 \rightarrow T=1)$ and ${ }^{24} \mathrm{Mg}$, and by $50 \%$ in ${ }^{22} N e(T=1 \rightarrow T=2)$. The summed orbital isovector $M 1$ strength $\left(B_{l}\right)$ increases by $20 \%$ and $30 \%$ for ${ }^{20} N e$ and ${ }^{22} N e$ respectively, but decreases by $4 \%$ for ${ }^{24} \mathrm{Mg}$. Finally the summed physical $\mathrm{B}(\mathrm{M} 1)$ 's increase by $20 \%$ to $40 \%$ for these nuclei $\left({ }^{20} \mathrm{Ne},{ }^{22} \mathrm{Ne}\right.$ and ${ }^{24} \mathrm{Mg}$ ). This overall increasing trend in the strength of the $B(M 1)$ 's is welcome because our calculations (especially with $x=1 y=1)$ typically underestimate the experimental data there.

However, if we look at the summed strength to the first 10 states, there is a much bigger change in going from $x=1.5 y=0.5$ to $x=1.5 y=0.5 \Delta \epsilon_{s}=2 \mathrm{MeV}$. This can be seen from tables III and IV. In particular, for ${ }^{24} \mathrm{Mg}$, the value of $B_{\sigma}$ to the first 10 states (and this more or less covers the range of experimental activity) changes from $1.79 \mu_{N}^{2}$ to $3.38 \mu_{N}^{2}$. This brings us close to the experimental value of $5.86 \mu_{N}^{2}$. The increase in $B_{\sigma}$ when we increase the $1 s$ single-particle energy can be easily understood. For a pure $(1 s)^{n}$ ground state configuration there can be no $M 1$ excitations. Thus, for say ${ }^{20} N e$, when we increase $\Delta \epsilon_{s}$, the occupation of $1 s$ goes down and the occupation of $0 d$ goes up. Thus there will be an increase in $B_{\sigma}$.

One can also see this from the energy-weighted sum rule of Kurath [13]. He assumed that the most important part of the Hamiltonian for $M 1$ spin transitions is the one-body spin-orbit interaction $-a \vec{l} \cdot \vec{s}$. Following a discussion of Zamick, Abbas and Halemane [14, the isovector $M 1$ operator can be written as $\sqrt{3 / 16 \pi}\left[-j_{i} \tau_{i}+\left(g_{n}-g_{p}+1\right) s_{i} \tau_{i}\right]$ where $\vec{j}=\vec{l}+\vec{s}, g_{n}=-3.836 \mu_{N}$ and $g_{l}=5.586 \mu_{N}$. Neglecting the $j_{i} \tau_{i}$ term, the energy weighted sum rule of Kurath [13] obtained by the double-commutator method is:

$$
\sum\left(E_{n}-E_{0}\right) B(M 1) \uparrow=\frac{3}{32 \pi} a\left(g_{n}-g_{p}+1\right)^{2} \sum_{l}\left[\ln _{l+1 / 2}-(l+1) n_{l-1 / 2}\right]
$$

where $n_{j}$ is the occupation of the $j$ orbit. Clearly if $l=0$ there will be no contribution. For the $s-d$ shell, the relevant factor is $\left(2 n_{d 5 / 2}-3 n_{d 3 / 2}\right)$.

For the $N=Z$ even-even nuclei in the second half of the shell, the effect of raising the $1 s$ level by $2 \mathrm{MeV}$ is less dramatic, perhaps due to the possible reasons noted above. In ${ }^{32} S$ and ${ }^{36} A r$ the physical $B(M 1)$ sums decrease by $5 \%$ and $7 \%$ respectively, the orbital $B(M 1)$ 's increase by $4 \%$ and $8 \%$ and the spin $B(M 1)$ 's decrease by $5 \%$ in ${ }^{36} A r$ and by $9 \%$ in ${ }^{32} S$.

In Table $\mathrm{V}$, and for the $x=1.5 y=0.5$ interaction, we can see the effects of raising the $1 \mathrm{~s}$ by $2 \mathrm{MeV}$ above the $0 \mathrm{~d}$ 
level on the summed $B(E 2)$ 's (from the $0^{+}$ground state to up to $5002^{+}$states). For the isoscalar transitions from the $J=2^{+} T=0$ (which dominate by an order of magnitude), the effect is a decrease in the first half of the shell (by about $15 \%$ in ${ }^{20} \mathrm{Ne}, 13 \%$ in the $T=1 \rightarrow T=1$ transitions in $22 \mathrm{Ne}$, and by $2 \%$ in ${ }^{24} \mathrm{Mg}$ ) and an increase in the second half of the shell (by $26 \%$ for ${ }^{32} S$ and by $10 \%$ for ${ }^{36} \mathrm{Ar}$ ). For the weaker isovector transitions, the effect is smaller; it is usually a decrease (by $11 \%$ in $20 N e, 4 \%$ for $T=1 \rightarrow T=2$ in ${ }^{22} N e, 4 \%$ for ${ }^{32} S$ and $7 \%$ in ${ }^{36} \mathrm{Ar}$ ) except for a $3 \%$ increase in ${ }^{24} \mathrm{Mg}$.

In all the five nuclei in Table $\mathrm{V}$, the isoscalar $B(E 2)$ sum over all the states is dominated by the $B(E 2)$ from the ground state to the lowest $J=2^{+} T=0$ state; that state contributes between about $75 \%$ and $90 \%$ of the total. By contrast, there is less dominance of a single state in the isovector $B(E 2)$ sum (from the ground state to the $J=2^{+} T=1$ states $)$. There the state with the strongest transition contributes about $20 \%\left({ }^{24} \mathrm{Mg}\right)$ to $60 \%\left({ }^{32} S\right)$.

\section{DISCUSSION OF INDIVIDUAL LEVELS -COMPARISON WITH EXPERIMENT}

We now proceed with a more systematic discussion of individual levels and a comparison with experiment. This will involve information contained in Tables VIII up to XXVI.

\section{A. ${ }^{8} \mathrm{Be}$}

In ${ }^{8} \mathrm{Be}$, there is a strong $M 1$ transition to the ground state from a $J=1^{+} T=1$ state at $17.6 \mathrm{MeV}$ from which we can infer that $B(M 1) \uparrow=0.8 \mu_{N}^{2}$. It is tempting to associate this $17.6 \mathrm{MeV}$ state with a scissors mode excitation. It is of interest to note that this is the only excited state of ${ }^{8} B e$ that communicates with the ground state i.e. has an observed branch to it. The $2^{+}$-to-ground $E 2$ is not observed because the $2^{+}$state quickly decays into two alpha particles. We expect ${ }^{8} B e$ to be a strongly deformed nucleus.

We find that for $(x=1, y=1)$ the strongest $B(M 1) \uparrow$ to a $1^{+} T=1$ state is to the lowest calculated state at $13.73 \mathrm{MeV}$ with $B(M 1) \uparrow=0.71 \mu_{N}^{2}$. The lowest $1^{+} T=0$ state is at a higher energy $(14.385 \mathrm{MeV})$. This inverse isospin ordering is in agreement with experiment. However the states come too low in energy compared with the experimental values (17.6 MeV and $18.5 \mathrm{MeV})$.

A spin-orbit analysis shows that the (calculated) state is not purely orbital: $B_{l}=0.26 \mu_{N}^{2}$ and $B_{\sigma}=0.15 \mu_{N}^{2}$. There is another state calculated to be nearly $3 \mathrm{MeV}$ above the $13.73 \mathrm{MeV}$ state with $B_{l}=0.23 \mu_{N}^{2}$, almost as much as for the lowest state. The value of $B_{\sigma}$ to this state is $0.053 \mu_{N}^{2}$. However, destructive interference between spin and orbit causes $B(M 1)$ to be negligible $\left(B(M 1)=0.065 \mu_{N}^{2}\right)$.

When we change from $x=1 y=1$ to $x=1.5 y=1, B_{l}$ changes very little, but $B_{\sigma}$ increases to $0.217 \mu_{N}^{2}$ and consequently $B(M 1)$ increases to $0.97 \mu_{N}^{2}$. If we finally change to $x=1.5, y=0.5$ i.e. increase the spin-orbit and decrease the tensor, the value of $B(M 1)$ increases slightly to $1.01 \mu_{N}^{2}$.

It should be noted that increasing the spin-orbit interaction does not increase the energy of the $J=1^{+}$state. From $x=1 y=1$ to $x=1.5 y=1$ the energy actually decreases from $13.73 \mathrm{MeV}$ to $13.06 \mathrm{MeV}$. This is contrary to the behaviour of a naive spherical model where the $1^{+}$state is pictured as a particle-hole state of the form $d_{3 / 2} d_{5 / 2}^{-1}$. The energy of such a state would clearly increase linearly with the spin-orbit strength $x$. However, since ${ }^{8} B e$ (and most of the other nuclei we will be considering here) are strongly deformed, such 'spherical' arguments do not hold water. This problem has been discussed by Zheng and Zamick [6]. Previous work on ${ }^{8} \mathrm{Be}$ and ${ }^{10} \mathrm{Be}$ by Fayache et. al. should be noted.

As seen from Table VI, there is also in ${ }^{8} \mathrm{Be}$ an 'invisible' state. For $x=1.5, y=0.5$ this state is $3.7 \mathrm{MeV}$ above the lowest strong state. The calculated values of $B_{l}, B_{\sigma}$ and $B(M 1)$ are $0.14,0.023$, and $0.050 \mu_{N}^{2}$ respectively. There is substantial orbital strength. Experimentally, such a state has not been seen. 
Studies of ${ }^{8} \mathrm{Be}$ and ${ }^{10} \mathrm{Be}$ were previously carried out by Fayache, Zamick and Sharma [15, 16]. The study was limited to the $x=1 y=1$ case, but the quadrupole-quadrupole interaction was also studied. With the $Q \cdot Q$ interaction, it can be shown that the the orbital strength $B_{l} \uparrow$ in ${ }^{8} B e$ is $\frac{2}{\pi} \mu_{N}^{2}=0.64 \mu_{N}^{2}$. This is very close to the results obtained in Table II (0.64 or $\left.0.67 \mu_{N}^{2}\right)$.

\section{B. ${ }^{10} \mathrm{Be}$}

In ${ }^{10} \mathrm{Be}$, there is no information on $M 1$ excitations. There is a strong $E 2$ from the ground state to the $2_{1}^{+}$state $B(E 2) \uparrow=52 e^{2} \mathrm{fm}^{4}$. Raman et.al. deduce from this a deformation parameter $\beta=1.13$ [17]. The experimental result is somewhat smaller than the isoscalar result of Table VII $\left(71 \mathrm{e}^{2} \mathrm{fm}^{4}\right)$. It should be remembered that the theoretical results were obtained with effective charges $e_{p}=1.5 e_{n}=0.5$. There is some evidence that in the $0 p$ shell, the effective charges should be somewhat smaller.

As shown by Fayache et.al. [16], with a $Q \cdot Q$ interaction one could obtain analytic results for the isovector orbital strength $B_{l}$ in ${ }^{10} \mathrm{Be}$. The results were:

$$
B_{l}\left(0^{+} T=1 \rightarrow 1^{+} T=1\right)=\frac{9}{32 \pi} \mu_{N}^{2}=0.0895 \mu_{N}^{2}
$$

and

$$
B_{l}\left(0^{+} T=1 \rightarrow 1^{+} T=2\right)=\frac{15}{32 \pi} \mu_{N}^{2}=0.1492 \mu_{N}^{2}
$$

The total orbital strength $(T=1+T=2)$ of $\frac{24}{32 \pi} \mu_{N}^{2}$ is only $3 / 8$ of the total orbital strength in ${ }^{8} B e$.

As seen in Table II, the results for isovector $B_{l}$ for all four cases of the $(x, y)$ interaction interaction seem to be in close accord with the analytic results of the $Q \cdot Q$ interaction above.

From Table $\mathrm{V}$ we note that the two lowest lying states fro $x=1 y=1$ are calculated to be at $6.14 \mathrm{MeV}$ and 7.68 $M e V$ with values of $B(M 1)$ of $1.110^{-2} \mu_{N}^{2}$ and $1.85 \mu_{N}^{2}$ respectively. These states have negligible orbital content -they are spin modes not orbital modes. The calculated $T=1$ and $T=2$ isovector orbital strengths (i.e. scissors modes) [16] are at much higher energies $\approx 19 \mathrm{MeV}$. With a $Q \cdot Q$ interaction, the $T=1$ and $T=2$ orbital strengths are degenerate. For $x=1.5 y=0.5$ we get two states with significant $B(M 1)$ strength. The energies are $8.45 \mathrm{MeV}$ and $9.26 \mathrm{MeV}$, and the values of $B(M 1) \uparrow$ are $0.77 \mu_{N}^{2}$ and $2.70 \mu_{N}^{2}$. The orbital content is still negligible.

In Table VI we list for ${ }^{10} \mathrm{Be}$ those states which contain most of the orbital strength. There are both $T=1$ and $T=2$ states, and for $x=1.5 y=0.5$ they range in energy from about $16 \mathrm{MeV}$ to $24 \mathrm{MeV}$. The orbital strength is at a much higher energy than the spin strength for ${ }^{10} \mathrm{Be}$.

$$
\text { C. }{ }^{12} \mathrm{C}
$$

As shown in Table VIII, there are two well-studied states in ${ }^{12} \mathrm{C}$ : the $1^{+} \mathrm{T}=0$ state at $12.71 \mathrm{MeV}$ and the $1^{+} T=1$ (isovector) state at $15.11 \mathrm{MeV}$. As mentioned in the introduction, the ratio $\left(\mid \frac{g_{s}(\text { vector })}{g_{s}(\text { scalar })}\right)^{2}=81$ so we expect the $1^{+} T=1$ state to be excited much more strongly than $T=0$ and indeed in the calculation this is the case. For $x=1 y=1$, we get

$$
\frac{B(M 1) \uparrow\left(\text { g.s. } \rightarrow 1^{+} T=1\right)}{B(M 1) \uparrow\left(g . s . \rightarrow 1^{+} T=0\right)}=\frac{0.856}{0.0026}=330
$$

The lowest calculated $1^{+}, T=1$ state in ${ }^{12} C$ is dominantly a spin state. For $x=1 y=1$ the values of $B(M 1)$ $\left(\right.$ in $\mu_{N}^{2}$ ) are $B_{l}=0.0314, B_{\sigma}=0.584$ and $B(M 1)=0.886$. For the other extreme $(x=1.5 y=0.5)$, we obtain 
$B_{l}=6.9110^{-5}, B_{\sigma}=2.571$ and $B(M 1)=2.54$. This analysis confirms the assumption made in testing the CVC theory by A. Richter at. al. -that the the $15.1 \mathrm{MeV}$ state is indeed an almost pure spin state. Only then can a relation between the isovector $M 1$ and a corresponding Gamow-Teller matrix element from beta decay be made.

Where there are orbital (scissors mode) excitations, the analysis is tricky. For $x=1.5 y=0.5$, the next excited $J=1^{+} T=1$ state is at $17.46 \mathrm{MeV}$, and it has $B_{l}=0.28 \mu_{N}^{2}$ and $B_{\sigma}=0.074 \mu_{N}^{2}$. But there is a large cancellation between spin and orbit so that $B(M 1)$ is only $0.068 \mu_{N}^{2}$. One would need in addition to electromagnetic excitation another probe e.g. proton scattering to unearth the substantial orbital strength at $20.19 \mathrm{MeV}\left(B_{l}=0.17 \mu_{N}^{2}\right.$, $\left.B_{\sigma}=0.0052 \mu_{N}^{2}, B(M 1)=0.113 \mu_{N}^{2}\right)$.

We now consider the $(x, y)$ dependence of $B(M 1)$. For the four values $(1,1),(1.5,1),(1,0.5)$ and $(1.5,0.5)$, the values of $B(M 1)$ (in $\mu_{N}^{2}$ ) are $0.89,1.89,1.12$ and 2.54 respectively. The experimental value is $2.63(8) \mu_{N}^{2}$ so we see that we need the full machinery (larger spin-orbit and weaker tensor interactions) to come even close to the large measured value. Again, as in ${ }^{8} B e$, the energies of the $1^{+}$states are somewhat too low, and changing $x$ and $y$ does not solve the problem. Thus for $x=1.5 y=0.5$ the $1^{+}$states are calculated to be at $11.8 \mathrm{MeV}(T=0)$ and $13.1 \mathrm{MeV}(T=1)$.

A comparison of experiment and theory for $B(E 2) \uparrow$ in ${ }^{12} C$ is made in Table IX. By far the largest $B(E 2)$ is to the first $2^{+}$state at $4.44 \mathrm{MeV}$. With the 'standard' effective charges $e_{p}=1.5 e_{n}=0.5$, the calculated values of $B(E 2)$ range from 70 to $80 e^{2} \mathrm{fm}^{4}$-they are not very sensitive to variations in $x$ and $y$. The experimental value is $40 e^{2} \mathrm{fm}^{4}$. It appears from the results here and in ${ }^{8} B e$ that the effective charges in the $0 p$ shell should be somewhat smaller than the standard value. For ${ }^{12} C$, and for all even-even nuclei considered here, the $B(E 2) T=0 \rightarrow T=0$ is proportional to $\left(e_{p}+e_{n}\right)^{2}$ and the $B(E 2) T=0 \rightarrow T=1$ to $\left(e_{p}-e_{n}\right)^{2}$.

\section{D. ${ }^{20} \mathrm{Ne}$}

The experimental data for ${ }^{20} \mathrm{Ne}$ is contained in Tables X and XI. In ${ }^{20} N e$ there is a strong $M 1$ excitation to a $J=1^{+} T=1$ state at $11.26 \mathrm{MeV}$. The experimental values to this state $\left(\right.$ in $\left.\mu_{N}^{2}\right)$ are $B_{l}=0.52(15), B_{\sigma}=0.49(6)$ and $B(M 1)=2.02(36)$. The calculated values of $B(M 1)$ for $x=1 y=1$ are $B_{l}=0.43, B_{\sigma}=0.06$ and $B(M 1)=0.83$. For $x=1.5 y=0.5$, we get $B_{l}=0.47, B_{\sigma}=0.35$ and $B(M 1)=1.63$. We thus get substantially better values of $B_{\sigma}$ and hence of $B(M 1)$ when we increase the spin-orbit interaction and decrease the tensor.

But, as mentioned previously, we appear to get the 'correct' spin-orbit splitting in ${ }^{17} O$ with a value of the spinorbit strength $x=1$. This gives a $d_{3 / 2}-d_{5 / 2}$ single-particle splitting of $5.2 \mathrm{MeV}$. For $x=1.5$ the value would be $5.2 \times 1.5=7.8 \mathrm{MeV}$. Thus the $M 1$ 's in ${ }^{20} \mathrm{Ne}$ (and in other $s-d$ shell nuclei as well) act as if the spin-orbit splitting is much larger than what is obtained from energy splittings for $A=17$.

When we further move the $1 s$ level up by $2 \mathrm{MeV}\left(\Delta \epsilon_{s}=2 \mathrm{MeV}\right)$ relative to $0 d$, the values of $B_{l}, B_{\sigma}$ and $B(M 1)$ to the strongest state (calculated to be at $9.76 \mathrm{MeV}$ ) become (in units of $\mu_{N}^{2}$ ) 0.51, 0.62 and 2.26 respectively. We see that $B_{\sigma}$ and hence $B(M 1)$ increase and indeed, for $B(M 1)$, we are within the errors bars of the experiment.

The dominant $E 2$ strength goes to the $2_{1}^{+}$state at $1.63 \mathrm{MeV}$. The measured $B(E 2) \uparrow$ is $340(22) e^{2} \mathrm{fm}^{4}$ whilst the calculated values (with $e_{p}=1.5, e_{n}=0.5$ ) are about $300 e^{2} \mathrm{fm}^{4}$-in good agreement. As in ${ }^{12} C$, there is very little sensitivity of $B(E 2)$ to $x$ and $y$. This could be understood if the extreme $L S$ coupling limit was valid. If the ground state is $L=0 S=0$ and the $2^{+}$state $L=2 S=0$ then the tensor and spin-orbit interactions would have no effect -the expectation values will vanish for $S=0$ states. With $\Delta \epsilon_{s}=2 \mathrm{MeV}$, the $B(E 2)$ to the $2_{1}^{+}$state decreases to 265 $e^{2} \mathrm{fm}^{4}$. There is some loss of collectivity when the $1 \mathrm{~s}$ level is raised in energy. 
Experimentally (see Table XII), the $B(M 1)$ 's in ${ }^{22} N e$ are as follows: $0.43(17) \mu_{N}^{2}$ to a state at $5.33 \mathrm{MeV}, 1.36(56)$ $\mu_{N}^{2}$ to a state at $6.85 \mathrm{MeV}$ and $1.80(7)$ to a state at $9.18 \mathrm{MeV}$. The summed experimental strength to the three states is $3.59 \mu_{N}^{2}$. For the four $(x, y)$ values we get the sum to be $1.62 \mu_{N}^{2}, 2.76 \mu_{N}^{2}, 1.96 \mu_{N}^{2}$ and $3.38 \mu_{N}^{2}$. We clearly get better results when $x=1.5$ (second and fourth cases), and we get the best results with $x=1.5, y=0.5$. In a later section, we shall see that even better results are obtained by increasing the $1 \mathrm{~s}$ level $\left(\Delta \epsilon_{s}=2 \mathrm{MeV}\right)$.

In the absence of a spin-orbit interaction, the nucleus ${ }^{22} \mathrm{Ne}$ is triaxial in a Hartree-Fock calculation e.g. with a Skyrme interaction. Introducing a spin-orbit interaction tends to make this and all other nuclei axially symmetric. Depending on what parameters are used, the calculation can go either way -triaxial or axially symmetric.

We can determine the degree of triaxiality by looking at $B(E 2) \uparrow$ from the ground state. In the axial case, the lowest $2^{+}$state will be a member of a $K=0$ ground state band and the next one of a different band with $K=2$. Thus $B(E 2) 0_{1} \rightarrow 2_{1}$ will be much stronger than $B(E 2) 0_{1} \rightarrow 2_{2}$. In the triaxial case, there will be much $K$ mixing and we can get strong $B(E 2)$ 's to both the $2_{1}$ and $2_{2}$ states.

Experimentally, as shown in Table XIII, the $B(E 2)$ to the first $2^{+}$state is much larger than to the second. The respective values are $300 e^{2} \mathrm{fm}^{4}$ and $12 e^{2} \mathrm{fm}^{4}$. These results favor axial symmetry for ${ }^{22} \mathrm{Ne}$.

In the shell model calculations with the $(x, y)$ interaction, we also see a strong dependence of the ratio $B(E 2)_{2}$ to $B(E 2)_{1}$ with the strength of the spin-orbit interaction, and indeed the results are consistent with those of Hartree-Fock calculations.

For $x=1$ (i.e. the free-space spin-orbit interaction) and $y=1$, we get strong $B(E 2)$ 's to both the first and second $2^{+}$states. The respective values are $199 e^{2} \mathrm{fm}^{4}$ and $113 \mathrm{e}^{2} \mathrm{fm}^{4}$. When we increase $x$ to 1.5 (keeping $y=1$ ) the second $B(E 2)$ shrinks considerably. The values are now $B(E 2)_{1}=289 e^{2} \mathrm{fm}^{4}$ and $B(E 2)_{2}=7.5 \mathrm{e}^{2} \mathrm{fm}^{4}$.

Thus the $B(E 2)$ data, just like the $M 1$ data, favour a larger spin-orbit interaction than in free space. Thus we have the interesting observation that the absence of triaxiality throughout the periodic table may be due to the fact that the spin-orbit interaction in a nucleus gets enhanced by medium modifications. As mentioned in the introduction, there are several models for such a modification -a Dirac effective mass in the Walecka model, and three-body interactions due to meson degrees of freedom in the Argonne-Illinois model.

\section{F. ${ }^{24} M g$}

In ${ }^{24} \mathrm{Mg}$ we have the $\left(e, e^{\prime}\right)$ results of Richter et. al. [3] as shown in Table XIV. They obtain a summed $B(M 1)$ of $4.85(36) \mu_{N}^{2}$ up to a maximum excitation energy $E_{\text {max }}=11.4 \mathrm{MeV}$, and the sum increases to $5.84(40) \mu_{N}^{2}$ for $E_{\max }=15 \mathrm{MeV}$.

In our calculation the summed $B(M 1)$ strength to all states in the $s d$ shell for the four values of $(x, y)$ are 3.78 , $4.95,3.87$ and $5.43 \mu_{N}^{2}$. The sums for the first 10 states (which have maximum excitation energies which vary from 15 to $16 \mathrm{MeV}$ i.e. in the same ballpark as the experiment) are smaller: 2.80, 3.28, 2.90 and $3.45 \mu_{N}^{2}$. We thus find that the experimental sum is larger than the theoretical sum by a factor $\frac{e x p}{\text { free }}=\frac{5.43}{3.45}=1.57$. The USD interaction of Wildenthal also gives a ratio bigger than one but it is much smaller than ours: 1.11(8).

However, with $\Delta \epsilon_{s}=2 \mathrm{MeV}$ in the $x=1.5 \mathrm{y}=0.5$ case, the situation improves a great deal. The total sum increases to $6.44 \mu_{N}^{2}$, and the sum to the first ten states increases to $4.58 \mu_{N}^{2}$.

Experimentally, two low-lying states absorb most of the $M 1$ strength. We have $B(M 1) \uparrow=1.5 \mu_{N}^{2}$ to a $9.96 \mathrm{MeV}$ state and $2.5 \mu_{N}^{2}$ to a $10.71 \mathrm{MeV}$ state. In the calculation however, for say $(x, y)=(1.5,0.5)$, the values of $B(M 1)$ greater than $0.3 \mu_{N}^{2}$ are $2.11 \mu_{N}^{2}$ to a $9.32 \mathrm{MeV}$ state and $0.64 \mu_{N}^{2}$ to a $9.47 \mathrm{MeV}$ state. Since in the shell model 
calculation these two states are nearly degenerate, it is hard to get the strengths just right. The experimental summed strength to the two states is $4 \mu_{N}^{2}$ whilst the calculation gives $2.75 \mu_{N}^{2}$.

In ${ }^{24} \mathrm{Mg}$ we also have results for $B_{\sigma}$ from proton inelastic scattering by Crawley et. al. [18] in $\left(p, p^{\prime}\right)$ reactions as shown in Table XV (see also Table XVI). The summed experimental strength for states ranging in energy from 8.865 to $14.267 \mathrm{MeV}$ is $5.86 \mu_{N}^{2}$. The first two $1^{+}$states at 8.865 and 9.820 are weakly excited: $B_{\sigma}=0.042 \pm 0.011$ and $0.260 \pm 0.040 \mu_{N}^{2}$ respectively. The next two states at 9.962 and $10.711 \mathrm{MeV}$ are strongly excited: $B_{\sigma}=1.160 \pm 0.180$ and $3.180 \pm 0.300 \mu_{N}^{2}$ respectively. The summed experimental strength for the above-mentioned energy range is 5.862 $\mu_{N}^{2}$.

Theoretically, the entire summed strength of $B_{\sigma}$ for the four values of $(x, y)$ are respectively $1.73,3.35,1.94$ and $4.04 \mu_{N}^{2}$. For the first ten states we get (for $\left.x=1.5, y=0.5\right) \sum B_{\sigma}=1.79 \mu_{N}^{2}$. This is considerably smaller than the experimental sum. But with $\Delta \epsilon_{s}=2 M e V$ the total $B_{\sigma}$ sum is $5.45 \mu_{N}^{2}$ and to the first ten states $3.38 \mu_{N}^{2}$.

As to individual levels and for $x=1.5 y=0.5$, we get nearly degenerate states at 9.325 and $9.466 \mathrm{MeV}$ with $B_{\sigma}=1.02$ and $0.27 \mu_{N}^{2}$ i.e. a summed strength of $1.29 \mu_{N}^{2}$. The summed experimental strength $\sum B_{\sigma}$ for the two strong states is $4.34 \mu_{N}^{2}$. With $\Delta \epsilon_{s}=2 M e V$ the corresponding summed $B_{\sigma}$ strength is $2.41 \mu_{N}^{2}$, somewhat closer to experiment.

$$
\text { G. }{ }^{28} S i
$$

From the work of Lüttge et. al. 19] as shown in Table XVIII, the summed B(M1) $\uparrow$ strength in ${ }^{28} S i$ up to an excitation energy of $18 \mathrm{MeV}$ is $7.53 \mu_{N}^{2}$. There is considerable fragmentation with the three strongest states at energies of 10.901, 11.445 and $12.331 \mathrm{MeV}$ having $B(M 1)$ 's of $0.90 \pm 0.02 \mu_{N}^{2}, 4.42 \pm 0.20 \mu_{N}^{2}$ and $0.87 \pm 0.06 \mu_{N}^{2}$ respectively. From the work of Crawley et. al. (Table XIX), the summed $B_{\sigma}$ strength up to an excitation energy of $15.8 \mathrm{MeV}$ is $9.51 \mu_{N}^{2}$. The $B_{\sigma}$ strength to the strongest state at $11.45 \mathrm{MeV}$ is $3.32 \pm 0.24 \mu_{N}^{2}$ as determined by Crawley et. al. 18.

For $x=1 y=1$, our summed $B(M 1)_{\text {isovector }}$ is $4.44 \mu_{N}^{2}$ with $\sum B_{l}=2.24 \mu_{N}^{2}$ and $\sum B_{\sigma}=2.39 \mu_{N}^{2}$. Our result is significantly less than experiment. The experimental $B(M 1)$ sum to the first ten states is $7.35 \mu_{N}^{2}$ whereas our sum is only $2.93 \mu_{N}^{2}$. The calculated $B(M 1)$ strength to individual states for $x=1 y=1$ are $1.35,0.49,0.39$ and $0.35 \mu_{N}^{2}$ at $10.31,11.78,12.97$ and $14.54 \mathrm{MeV}$ respectively. We seem to be missing the very strong state with an experimental $B(M 1)$ of $4.42 \mu_{N}^{2}$.

The results are dramatically improved when we go to $x=1.5$. The value of $B(M 1)$ to the single strongest state for the cases $(x, y)=(1,1)$ and $(1.5,0.5)$ are respectively $1.35 \mu_{N}^{2}$ and $3.90 \mu_{N}^{2}$. There is almost a factor of three increase and we come close to the experimental value of $4.42 \mu_{N}^{2}$. The corresponding change in $B_{\sigma}$ shown in Tables XIX, XX and XXI is from $0.86 \mu_{N}^{2}$ to $2.26 \mu_{N}^{2}$, in much better agreement but still lower than the experimental value. From $(x, y)=(1,1)$ to $(1.5,0.5)$ the total summed strength (Table II) for $B(M 1)$ changes from 4.44 to $6.38 \mu_{N}^{2}$, whilst the total summed $B_{\sigma}$ strength changes from 2.39 to $6.79 \mu_{N}^{2}$. If we limit the sum to the first ten states (Table III), the change in $B_{\sigma}$ is from 1.29 to $4.76 \mu_{N}^{2}$. In the latter case, $B_{\sigma}$ increases by a factor of 3.7 .

We nest consider the case $(x, y)=(1.5,0.5)$ with $\Delta \epsilon_{s}=2 M e V$. The values of $B(M 1)$ and $B_{\sigma}$ to the single strongest state (calculated to be at $10.03 \mathrm{MeV}$ ) are $4.61 \mu_{N}^{2}$ and $4.26 \mu_{N}^{2}$ respectively. The total summed strength for $B(M 1)$ and $B_{\sigma}$ are now increased to 8.94 and $9.04 \mu_{N}^{2}$, whilst to the first ten states the sums are 7.08 and $7.24 \mu_{N}^{2}$.

In contrast to $B_{\sigma}$, there is a substantial decrease in $B_{l}$ when we go from $(x, y)=(1,1)$ to $(1.5,0.5)$. The respective values for the total $B_{l}$ strengths are $2.24 \mu_{N}^{2}$ and $1.46 \mu_{N}^{2}$ respectively. With $(x, y)=(1.5,0.5)$ and $\Delta \epsilon_{s}=2 M e V$, there is a further decrease to $1.36 \mu_{N}^{2}$. 


\section{H. ${ }^{32} S$}

The results are shown in Table XXII. The most clearcut data in ${ }^{32} S$ appears for $B_{\sigma}$ by Crawley et. al. [18]. The summed strength is $10.75 \mu_{N}^{2}$ and the strongest excited states and the corresponding $\left(B_{\sigma}\right.$ in $\left.\mu_{N}^{2}\right)$ are: $8.13 \mathrm{MeV}(1.46$ $\pm 0.19), 11.13 \mathrm{MeV}(4.08 \pm 0.53)$ and $11.63 \mathrm{MeV}(2.38 \pm 0.35)$. For $x=1.5 y=0.5$ our summed $B_{\sigma}$ strength from table XXIV is $11.16 \mu_{N}^{2}$ to the first ten states and $12.03 \mu_{N}^{2}$ to all states. The agreement with experiment is reasonable. Our strongest state, however, has a $B_{\sigma}$ strength of $5.98 \mu_{N}^{2}$ at $11.21 \mathrm{MeV}$.

When for the case $x=1.5 y=0.5$ case we also use $\Delta \epsilon_{s}=2 \mathrm{MeV}$, the value of $B_{\sigma}$ to the first ten states drops somewhat to $9.45 \mu_{N}^{2}$, and the total sum also drops to $10.91 \mu_{N}^{2}$. The strongest state calculated to be at $10.25 \mathrm{MeV}$ now has $B_{\sigma}=4.20 \mu_{N}^{2}$, a significant drop from $5.98 \mu_{N}^{2}$.

Note that this is the first time we get a drop in $B_{\sigma}$ when $\Delta \epsilon_{s}=2 \mathrm{MeV}$ is used; for ${ }^{20} \mathrm{Ne},{ }^{22} \mathrm{Ne},{ }^{24} \mathrm{Mg}$ and ${ }^{28} \mathrm{Si}$ there was a rise. Note further that our results with this drop are in better agreement with experiment.

The $B(M 1)$ data is a bit complicated. In Table XXII from the results reported in the paper of Crawley et. al. 18] only a few states are seen, with a summed strength of $5.43 \mu_{N}^{2}$. However, there is other data by Petraitis et. al. 20] which shows strength to many other states. The summed Peraitis strength is $4.21 \mu_{N}^{2}$. However, he does not have the state at $8.13 \mathrm{MeV}\left(B(M 1) \uparrow=1.14 \pm 0.18 \mu_{N}^{2}\right)$, and disagrees on the $B(M 1)$ to the $11.13 \mathrm{MeV}$ state for which Petraitis gets $1.24 \pm 0.13 \mu_{N}^{2}$ but ref. [18] reports $2.40 \pm 0.22 \mu_{N}^{2}$.

We feel the best estimate at the moment is to take all the $M 1$ states of ref. [18] and add to that the strength of Petraitis et. al. to states not found in ref. [18]. Thus we get schematically strength reported in ref. [18] plus (strength reported by Petraitis et. al. minus strength to states at $9.66 \mathrm{MeV}, 11.16 \mathrm{MeV}$ and $11.85 \mathrm{MeV}$ ). When all this is done, we get a summed $B(M 1)$ strength of $7.61 \mu_{N}^{2}$. Our calculated $B(M 1)$ strength (with $x=1.5 y=0.5$ ) is 10.98 $\mu_{N}^{2}$ to all states. With $\Delta \epsilon_{s}=2 \mathrm{MeV}$, this drops to $10.12 \mu_{N}^{2}$, whilst the summed strength to the first ten states (i.e. up to $15.1 \mathrm{MeV}$ excitation energy) is $8.81 \mu_{N}^{2}$.

It is difficult to draw a definitive conclusion for ${ }^{32} S$ because two sets of $M 1$ data are not in accord. From the $B_{\sigma}$ data, it appears that we need an enlarged spin-orbit interaction. For $x=1 y=1$ the sum $B_{\sigma}$ is only $2.73 \mu_{N}^{2}$ whereas for $x=1.5 y=0.5$ it is $12.03 \mu_{N}^{2}$, closer to the experimental sum of $10.75 \mu_{N}^{2}$. However, the $M 1$ data supports a somewhat weaker spin-orbit and/or a stronger tensor interaction. Again, from Table II, the case $x=1.5 y=1$ gives results of $8.69 \mu_{N}^{2}$ for $B(M 1)$ and $8.52 \mu_{N}^{2}$ for $B_{\sigma}$.

It is significant that raising the $1 s$ level relative to $0 d$ causes a rise in $B_{\sigma}$ for ${ }^{20} \mathrm{Ne},{ }^{24} \mathrm{Mg}$ and ${ }^{28} \mathrm{Si}$, whilst for ${ }^{32} \mathrm{~S}$ and as we shall see ${ }^{36} \mathrm{Ar}$ it causes a decrease in $B_{\sigma}$.

$$
\text { I. }{ }^{36} \mathrm{Ar}
$$

For ${ }^{36} A r$, we refer to Tables XXVII, XXVIII and XXIX. Because of the large error bars on the $\left(e, e^{\prime}\right)$ experiment, we will concern ourselves here with the summed strength only. The measured value is $2.65 \pm 0.1 \mu_{N}^{2}$.

The total summed strengths for the four values of $(x, y)$ are $3.35,5.19,3.72,5.71 \mu_{N}^{2}$ respectively. The sums to the first ten states are 2.98, 4.82, 3.58 and $5.50 \mu_{N}^{2}$ respectively. They are almost the same as the complete sums.

The Foltz work [21] contains a theoretical discussion of shell model calculations using the Wildenthal interaction [甘1 Even with this phenomenological interaction there is a difficulty in the sense that the theory predicts a strong excitation of $B(M 1)=1.3 \mu_{N}^{2}$ at $10.3 \mathrm{MeV}$, whereas experimentally there is very strong fragmentation. Also the theory with bare operators predicts too large a summed strength $\left(5.13 \mu_{N}^{2}\right)$ for all the strength, and $4.73 \mu_{N}^{2}$ for the strength below $15 \mathrm{MeV}$. However, using effective operators, whose values are consistent with the fundamental calculations of Towner and Khanna [22], the summed strength is reduced and is closer to experiment. The above two numbers now become $3.54 \mu_{N}^{2}$ to all states and $2.86 \mu_{N}^{2}$ to states below $15 \mathrm{MeV}$. 
Thus the results we obtain with $x=1.5 y=0.5$, although still too large, are not so different from those obtained in the theoretical discussion of Foltz et. al. [21]. As seen in Tables III and IV, we get summed strengths of $5.71 \mu_{N}^{2}$ to all states and $5.50 \mu_{N}^{2}$ to the first ten states. With $\Delta \epsilon_{s}=2 \mathrm{MeV}$, the corresponding numbers are 5.48 and $5.10 \mu_{N}^{2}$.

$$
\text { J. }{ }^{44} \mathrm{Ti}
$$

There are no $M 1$ data for ${ }^{44} \mathrm{Ti}$ so we concentrate on $B(E 2)$ 's -these are shown in Tables XXX and XXXI. The experimental value of $B(E 2) \uparrow$ is $540(140) e^{2} \mathrm{fm}^{4}$ but the calculated values (with $e_{p}=1.5 e_{n}=0.5$ ) are much larger -from 908 to $925 \mathrm{e}^{2} \mathrm{fm}^{4}$ for various combinations of $x$ and $y$.

The probable reason for the calculated over-collectivity is that the $1 p$ level is too low in energy relative to $0 f$. This is confirmed in calculations in which we raise the $1 p$ centroid by $2 \mathrm{MeV}$ relative to the $0 f$ centroid. In that case, the value of $B(E 2) \uparrow$ is reduced to $710 e^{2} \mathrm{fm}^{4}$ (for $x=1.5 y=0.5$ ).

\section{CLOSING REMARKS}

In this work we simulate a realistic $G$-matrix by the interaction $V_{c}(r)+x V_{s . o}+y V_{t}$ where $x=1, y=1$ gives us matrix elements close to those obtained in a non-relativistic approach. We must emphasize at first that we use the same interaction to calculate single-particle energies as is used for the valence interactions. In this, we differ from most other calculations in which a hybrid approach is taken -most often the single-particle energies are taken from experiment. We feel however that one is not really testing the interaction. Later we relax this condition somewhat by shifting the $1 s$ single-particle energy relative to $0 d$.

In an open-shell nucleus the tensor interaction has the opposite effect of the spin-orbit interaction [12]. That is to say when the strength of the tensor interaction is decreased, the summed spin strength $\sum B_{\sigma}$ increases. There seems to be a phenomenological need for a weaker tensor interaction in a nucleus than in free space, and several arguments have been proposed to justify this. At the simplest level, there is the 'self-weakening mechanism' 10,24]. This simply means that the effects of higher-shell admixtures (i.e. $\Delta N=2$ excitations and beyond) can be simulated in the valence space by using a weaker tensor interaction. This is a purely nuclear structure justification, and the calculations for these admixtures can be carried out either perturbatively or in large shell model spaces. There is also the 'universal scaling' idea of Brown and Rho [9] that all mesons, except for the pion, are less massive inside a nuclear medium than in free space. This leads to a larger range for the contribution to the tensor interaction of $\rho$-exchange. This then causes a greater cancellation between the $\rho$ - and $\pi$-exchange contributions to the tensor interaction, leading to the overall weakening of the tensor interaction.

In our calculations, $B_{\sigma}$ does indeed increase when the tensor interaction is cut in half. Although the effect is not so large as increasing the spin-orbit interaction (from $x=1$ to $x=1.5$ ), overall the effects seem beneficial with the possible exception of ${ }^{36} \mathrm{Ar}$.

It should be further noted that the tensor interaction seems to have very little effect on the $B(E 2) 0_{1} \rightarrow 2_{1}$. This is undoubtedly because for these states one is approaching the $L S$ limit, and the wavefunctions are dominantly $S=0$. The tensor interaction vanishes for $S=0$ states. To our mind, this means that one can control $B_{\sigma}$ to some extent without affecting the low-lying spectroscopy. Note that for the $N=Z$ nuclei considered here, the isovector transition $B_{\sigma}(J=0 T=0 \rightarrow J=1 T=1)$ is proportional to the Gamow-Teller transition $B(G T)$. It has often been said that $B(G T)$ has to be quenched and there are even more specific statements that the $G T$ coupling constant which is 1.251

in free space should be equal to unity inside the nucleus. Our calculations here cast some doubt on such detailed conclusions. First of all, the famous sum rule $B(G T)_{+}=3(N-Z)+B(G T)_{-}$puts no restrictions whatsoever on $B(G T)$ for an $N=Z$ nucleus- one only gets the result $B(G T)_{+}=B(G T)_{-}$which is simply isospin conservation. 
Secondly, we know that $B_{\sigma}$ isovector and $B(G T)$ will vanish in the $S U(4)$ limit for $N=Z$ nuclei. At the other limit of $j j$ coupling, $B_{\sigma}$ and $B(G T)$ are much too large. So it seems we can get almost any answer we want. Indeed, for $x=1 y=1$ we get too small a $B(G T)$ and if we really believed in this interaction we could conclude that $B(G T)$ had to be enhanced. On the other hand, if we had chosen $x=2 y=1$ (a very large spin-orbit interaction), we could conclude that $B(G T)$ had to be quenched. Perhaps a case can be made that the $3(N-Z)$ part of the sum rule is quenched but not the $B(G T)$ part.

On the other hand, in the work of Richter, Weiss, Hausser and Brown [3], the discussion of quenching pertains to the ratio of $B(M 1)_{\sigma}$ to $B(G T)$, and here one is on much firmer ground. By taking the ratio the uncertainties of the details of the nuclear interaction largely disappear, and the conclusion that $B(G T)$ is more quenched than $B(M 1)$ is certainly valid.

In the $0 p$ shell, where there is only one $l$ orbit, it is easier to draw conclusions. With $x=1 y=1$, we get a $B(M 1)$ of only $0.89 \mu_{N}^{2}$ to the $15.11 \mathrm{MeV}$ state in ${ }^{12} \mathrm{C}$-the experimental value is $2.63(8) \mu_{N}^{2}$. For $x=1.5 y=1$, the value is $1.89 \mu_{N}^{2}$, but only when we both increase the spin-orbit and decrease the tensor interaction $(x=1.5 y=0.5)$ do we get a $B(M 1)$ comparable with experiment $\left(2.55 \mu_{N}^{2}\right)$.

In the $s-d$ shell, things are somewhat more complicated by the fact that there are two $l$ orbits. Our initial calculation (in which the $0 d_{5 / 2}, 1 s_{1 / 2}$ and $0 d_{3 / 2}$ single-particle energies are calculated with the same $(x, y)$ interaction as is used in the valence space) puts the centroid of the $1 s$ level too low in energy relative to that of $0 d$. This has the effect of making $B_{\sigma}$ too small in the lower half of the $s-d$ shell e.g. ${ }^{20} \mathrm{Ne},{ }^{22} \mathrm{Ne},{ }^{24} \mathrm{Mg}$ and ${ }^{28} \mathrm{Si}$. By moving the $1 s$ level up by $2 \mathrm{MeV}$ we increase $B_{\sigma}$ significantly for these nuclei, and as a bonus, in the upper half of the $s-d$ shell i.e. ${ }^{32} S$ and ${ }^{36} \mathrm{Ar} B_{\sigma}$ gets slightly decreased, and this appears all to the good.

We find that for most nuclei, the non-relativistic $G$-matrix, as given by the parameters $x=1 y=1$ gives much too small values for the spin excitation rates $\left(B_{\sigma}\right)$. By increasing the two-body spin-orbit term from $x=1$ to $x=1.5$ we can greatly improve the situation. Microscopic justification for this came from the Dirac phenomenology in which a Dirac effective mass $m^{*}$ is introduced, and is said to arise from the exchange of $\sigma$ mesons between nucleons. The spin-orbit interaction gets increased by a factor $\frac{m}{m^{*}}$ (however $m^{*}$ is a function of $r$ and so the effect should be greater in the interior than on the surface of the nucleus). There is an alternate three-body approach [7] which also gives an enhanced spin-orbit splitting.

Lastly, we have here introduced a new topic -the cross-correlation between the spin and orbital parts of $B(M 1)$. In our calculations, we find that the the total sum $\sum B(M 1)$ is very close to $\sum B_{\sigma}+\sum B_{l}$, indicating that the sum of the cross terms nearly vanishes.

\section{ACKNOWLEDGEMENTS}

Y. Sharon received support on his sabbatical from Richard Stockton College of New Jersey. This work was supported by a D.O.E. grant DE-FG05-86ER-40299 and by the German Federal Ministry of Education, Research and Technology (BMBF) under contract No. 06DA6651. One of us (L.Z.) acknowledges support from the Alexander Von Humboldt Foundation. We thank Alex Brown for useful communications. 
[1] S. Cohen and D. Kurath, Nucl. Phys. 73,(1965)1.

[2] B.A. Brown and B.H. Wildenthal, Phys. Rev. C 27,(1983)1296.

[3] A. Richter, A. Weiss, O. Hausser and B.A. Brown, Phys. Rev. Lett. 65, (1990)2519.

[4] B.A. Brown and B.H. Wildenthal, Nucl. Phys. A 474, (1987)290.

[5] B.H. Wildenthal, in "Progress in Particle and Nuclear Physics", edited by D.H. Wilkinson (Pergamon, Oxford, 1984), Vol 11, p. 5; B.A. Brown and H. Wildenthal, Ann. Rev. Nucl.Part. Sci., 38, (1988)29.

[6] D.C. Zheng and L. Zamick, Annals of Physics 206, (1991)106.

[7] B.D. Serot and J.D. Walecka, Adv. in Nucl. Phys. 16, (1986)1.

[8] S.C. Pieper and V.R. Pandharipande, Phys. Rev. Lett. 70, (1993)2541.

[9] G.E. Brown and M. Rho, Phys. Rev. Lett. 66, (1991)2720; Phys. Lett. B237, (1990)3.

[10] L. Zamick, D.C. Zheng and M.S. Fayache, Phys. Rev. C 51, (1996)1253.

[11] B. A. Brown, A. Etchegoyen and W. D. M. Rae, The computer code OXBASH, MSU-NSCL report number 524, 1992.

[12] C.W. Wong, Nucl. Phys. A 108, (1968)481.

[13] D. Kurath, Phys. Rev. 130, (1963)525.

[14] L. Zamick, A. Abbas and T.R. Halemane, Phys. Lett. 103 B, (1981)87.

[15] M.S. Fayache and L. Zamick, Phys. Lett. B 338, (1994)421.

[16] M.S. Fayache, S. Shelly Sharma and L. Zamick, submitted to Annals of Physics.

[17] S. Raman, C.H. Malarkey, W.T. Milner, C.W. Nesta Jr. and P.H. Steltson, Atomic Data and Nuclear Data Tables 36, $(1987) 1$.

[18] G.M. Crawley et. al., Phys. Rev. C 39, (1989)311.

[19] C. Lüttge et. al., Phys. Rev C 53, (1996)127.

[20] M. Petraitis et. al., Phys. Rev. C 49, (1994)3000.

[21] C.W. Foltz, D.I. Sober, L.W. Fagg, H.D. Gräf, A. Richter, E. Spames and B. A. Brown, Phys. Rev. C 49 , (1994)1359.

[22] I.S. Towner and F.C. Khanna, Nucl. Phys. A 399, 334 (1983).

[23] B.A. Brown and B.H. Wildenthal, Phys. Rev. C 27, (1983)1296; Nucl. Phys. A 474, (1987)290.

[24] D.C. Zheng, L. Zamick, M. Fayache, and H. Müther, Ann. Phys. (NY), 230, (1994)118. 
TABLE I. Single-Particle Energies Calculated with the $(x, y)$ interaction $(x=1, y=1)$ in a small space $(0 \hbar \omega)$ and a large space $(0+2) \hbar \omega$.

\begin{tabular}{llll}
\hline \hline Nucleus & State & $0 \hbar \omega$ & $(0+2) \hbar \omega$ \\
\hline${ }^{5} \mathrm{He}$ & $3 / 2^{-}$ & 0 & 0 \\
& $1 / 2^{-}$ & 3.375 & 2.224 \\
\hline${ }^{15} \mathrm{O}$ & $1 / 2^{-}$ & 0 & 0 \\
& $3 / 2^{-}$ & 5.063 & 5.679 \\
\hline${ }^{17} \mathrm{O}$ & $1 / 2^{+}$ & 0 & 0 \\
& $5 / 2^{+}$ & 0.119 & 1.430 \\
& $3 / 2^{+}$ & 5.681 & 7.092 \\
${ }^{39} \mathrm{Ca}$ & $3 / 2^{+}$ & 0 & \\
& $1 / 2^{+}$ & 5.457 & \\
\hline${ }^{41} \mathrm{Ca}$ & $5 / 2^{+}$ & 6.973 & \\
& $3 / 2^{-}$ & 0 & \\
& $7 / 2^{-}$ & 0.940 & \\
& $1 / 2^{-}$ & 2.458 & \\
\hline \hline
\end{tabular}


TABLE II. The Summed isovector $M 1$ strength in $\mu_{N}^{2}$ for various strengths $(x, y)$ of the spin-orbit and tensor interaction

\begin{tabular}{llllll}
\hline \hline Nucleus & $x$ & $y$ & $B(M 1)$ & $B_{l}$ & $B_{\sigma}$ \\
\hline${ }^{8} B e$ & 1 & 1 & 1.05 & 0.67 & 0.38 \\
& 1.5 & 1 & 1.28 & 0.64 & 0.65 \\
& 1 & 0.5 & 1.06 & 0.67 & 0.41 \\
& 1.5 & 0.5 & 1.33 & 0.64 & 0.73 \\
\hline${ }^{10} B e$ & 1 & 1 & 2.09 & $0.111(0.025)$ & 1.62 \\
$T=1 \rightarrow T=1$ & 1.5 & 1 & 3.17 & $0.105(0.036)$ & 2.64 \\
& 1 & 0.5 & 2.46 & $0.111(0.028)$ & 2.03 \\
& 1.5 & 0.5 & 3.55 & $0.103(0.043)$ & 3.11
\end{tabular}

$\begin{array}{llllll}{ }^{10} \mathrm{Be} & 1 & 1 & 0.060 & 0.151 & 0.093\end{array}$

$T=1 \rightarrow T=2 \quad 1.5 \quad 1 \quad 0.029 \quad 0.144 \quad 0.189$

$\begin{array}{llllll}1 & 0.5 & 0.046 & 0.152 & 0.104\end{array}$

\begin{tabular}{llllll} 
& 1.5 & 0.5 & 0.050 & 0.142 & 0.246 \\
\hline${ }^{12} C$ & 1 & 1 & 1.42 & 0.60 & 0.91
\end{tabular}

$\begin{array}{lllll}1.5 & 1 & 2.29 & 0.50 & 2.07\end{array}$

$\begin{array}{lllll}1 & 0.5 & 1.58 & 0.59 & 1.13\end{array}$

\begin{tabular}{llllll} 
& 1.5 & 0.5 & 2.85 & 0.46 & 2.82 \\
\hline${ }^{20} \mathrm{Ne}$ & 1 & 1 & 1.53 & 0.95 & 0.59
\end{tabular}

$\begin{array}{lllll}1.5 & 1 & 2.34 & 1.09 & 1.28\end{array}$

$\begin{array}{lllll}1 & 0.5 & 1.57 & 0.96 & 0.63\end{array}$

$\begin{array}{lllll}1.5 & 0.5 & 2.54 & 1.10 & 1.49\end{array}$

\begin{tabular}{llllll}
$\Delta \epsilon_{s}=2 \mathrm{MeV}^{\mathrm{a}}$ & 1.5 & 0.5 & 3.50 & 1.39 & 2.21 \\
\hline${ }^{22} \mathrm{Ne}$ & 1 & 1 & 2.28 & $0.029(0.36)^{\mathrm{b}}$ & 1.81
\end{tabular}

$T=1 \rightarrow T=1 \quad 1.5 \quad 1 \quad 3.98 \quad 0.046(0.44) \quad 3.20$

$\begin{array}{lllll}1 & 0.5 & 2.51 & 0.030(0.37) & 2.02\end{array}$

$\begin{array}{lllll}1.5 & 0.5 & 4.51 & 0.054(0.46) & 3.69\end{array}$

$\begin{array}{llllll}\Delta \epsilon_{s}=2 M e V & 1.5 & 0.5 & 5.79 & 0.068(0.486) & 4.32\end{array}$

$\begin{array}{llllll}{ }^{22} \mathrm{Ne} & 1 & 1 & 0.34 & 0.27 & 0.16\end{array}$

$T=1 \rightarrow T=2 \quad 1.5 \quad 1 \quad 0.38 \quad 0.31 \quad 0.39$

$\begin{array}{lllll}1 & 0.5 & 0.32 & 0.28 & 0.17\end{array}$

$\begin{array}{lllll}1.5 & 0.5 & 0.41 & 0.32 & 0.51\end{array}$

\begin{tabular}{llllll}
$\Delta \epsilon_{s}=2 \mathrm{MeV}$ & 1.5 & 0.5 & 0.48 & 0.38 & 0.76 \\
\hline${ }^{24} \mathrm{Mg}$ & 1 & 1 & 3.78 & 2.08 & 1.73
\end{tabular}

$\begin{array}{lllll}1.5 & 1 & 4.95 & 1.75 & 3.35\end{array}$

$\begin{array}{lllll}1 & 0.5 & 3.87 & 2.02 & 1.94\end{array}$

$\begin{array}{lllll}1.5 & 0.5 & 5.43 & 1.69 & 4.04\end{array}$

\begin{tabular}{llllll}
$\Delta \epsilon_{s}=2 \mathrm{MeV}$ & 1.5 & 0.5 & 6.45 & 1.63 & 5.46 \\
\hline${ }^{28} \mathrm{Si}^{\mathrm{c}}$ & 1 & 1 & 4.44 & 2.24 & 2.39
\end{tabular}

$\begin{array}{lllll}1.5 & 1 & 6.38 & 1.60 & 5.33\end{array}$

$\begin{array}{lllll}1 & 0.5 & 4.59 & 2.06 & 2.79\end{array}$

$\begin{array}{lllll}1.5 & 0.5 & 7.54 & 1.46 & 6.97\end{array}$




\begin{tabular}{llllll}
$\Delta \epsilon_{s}=2 \mathrm{MeV}$ & 1.5 & 0.5 & 8.94 & 1.36 & 9.04 \\
\hline${ }^{32} S$ & 1 & 1 & 4.76 & 2.32 & 2.73 \\
& 1.5 & 1 & 8.69 & 1.57 & 8.52 \\
& 1 & 0.5 & 5.43 & 2.17 & 3.76 \\
$\Delta \epsilon_{s}=2 \mathrm{MeV}$ & 1.5 & 0.5 & 10.19 & 1.24 & 12.03 \\
${ }^{36} \mathrm{Ar}$ & 1 & 1 & 3.35 & 1.44 & 2.21 \\
& 1.5 & 1 & 5.19 & 0.95 & 2.25 \\
& 1 & 0.5 & 3.72 & 1.33 & 5.20 \\
& 1.5 & 0.5 & 5.71 & 0.87 & 2.87 \\
$\Delta \epsilon_{s}=2 \mathrm{MeV}$ & 1.5 & 0.5 & 5.48 & 0.90 & 6.05 \\
\hline${ }^{44} \mathrm{Ti}$ & 1 & 1 & 2.41 & 1.50 & 0.72 \\
\hline & 1.5 & 1 & 3.41 & 1.63 & 1.79 \\
& 1 & 0.5 & 2.42 & 1.47 & 0.94 \\
$\Delta \epsilon_{p}=2 \mathrm{MeV} V^{\mathrm{d}}$ & 1.5 & 0.5 & 3.64 & 1.64 & 2.04 \\
\hline \hline
\end{tabular}

${ }^{\mathrm{a}}$ The $1 s$ orbital is raised by $2 \mathrm{MeV}$ above the $0 d$ orbital

${ }^{\mathrm{b}}$ The first number in this column is the isoscalar summed strength; the second number (in parentheses) is the isovector summed strength.

${ }^{\mathrm{c}}$ For $(x, y)=(1,1)$ the sum is over the 500 lowest states, but for all the other $(x, y)$ pairs the sum is over the lowest 50 states only.

${ }^{\mathrm{d}}$ The $1 p$ orbital is raised by $2 M e V$ above the $0 f$ orbital 
TABLE III. Summed isovector $M 1$ strength (First Ten States and All States) for $x=1.5, y=0.5$

\begin{tabular}{|c|c|c|c|}
\hline${ }^{20} \mathrm{Ne}$ & $B(M 1)$ & $B_{l}$ & $B_{\sigma}$ \\
\hline 1st 10 States & 2.05 & 0.90 & 1.22 \\
\hline All States & 2.54 & 1.10 & 1.49 \\
\hline \multicolumn{4}{|c|}{${ }^{22} \mathrm{Ne} T=1 \rightarrow T=1$} \\
\hline 1st 10 States & 3.71 & 0.23 & 2.88 \\
\hline All States & 4.51 & 0.46 & 3.69 \\
\hline \multicolumn{4}{|c|}{${ }^{22} \mathrm{Ne} T=1 \rightarrow T=2$} \\
\hline 1st 10 States & 0.23 & 0.25 & 0.36 \\
\hline All States & 0.41 & 0.31 & 0.51 \\
\hline \multicolumn{4}{|l|}{${ }^{24} \mathrm{Mg}$} \\
\hline 1st 10 States & 3.45 & 0.94 & 1.79 \\
\hline All States & 5.43 & 1.68 & 4.03 \\
\hline \multicolumn{4}{|l|}{$\overline{28} \mathrm{Si}$} \\
\hline 1st 10 States & 5.30 & 0.66 & 4.76 \\
\hline All States & 7.54 & 1.46 & 6.97 \\
\hline \multicolumn{4}{|l|}{${ }^{32} S$} \\
\hline 1st 10 States & 10.13 & 0.53 & 11.16 \\
\hline All States & 10.98 & 1.15 & 12.03 \\
\hline \multicolumn{4}{|l|}{${ }^{36} A r$} \\
\hline 1st 10 States & 5.50 & 0.63 & 5.95 \\
\hline All States & 5.71 & 0.87 & 6.05 \\
\hline \multicolumn{4}{|l|}{$\overline{{ }^{44} \mathrm{Ti}}$} \\
\hline 1st 10 States & 2.73 & 1.18 & 1.10 \\
\hline All States & 3.64 & 1.64 & 2.04 \\
\hline
\end{tabular}


TABLE IV. Same as Table III $(x=1.5, y=0.5)$ but with $\Delta \epsilon_{s}=2 \mathrm{MeV}$ for the $s-d$ shell nuclei and $\Delta \epsilon_{p}=2 \mathrm{MeV}$ for ${ }^{44} \mathrm{Ti}$

\begin{tabular}{|c|c|c|c|}
\hline${ }^{20} \mathrm{Ne}$ & $B(M 1)$ & $B_{l}$ & $B_{\sigma}$ \\
\hline 1st 10 States & 3.39 & 1.25 & 2.09 \\
\hline All States & 3.50 & 1.39 & 2.21 \\
\hline \multicolumn{4}{|c|}{${ }^{22} N e T=1 \rightarrow T=1$} \\
\hline 1st 10 States & 5.20 & 0.25 & 4.18 \\
\hline All States & 5.79 & 0.49 & 4.80 \\
\hline \multicolumn{4}{|c|}{${ }^{22} \mathrm{Ne} T=1 \rightarrow T=2$} \\
\hline 1st 10 States & 0.36 & 0.29 & 0.67 \\
\hline All States & 0.48 & 0.38 & 0.76 \\
\hline \multicolumn{4}{|l|}{${ }^{24} \mathrm{Mg}$} \\
\hline 1st 10 States & 4.59 & 0.81 & 3.38 \\
\hline All States & 6.45 & 1.63 & 5.45 \\
\hline \multicolumn{4}{|l|}{${ }^{28} \mathrm{Si}$} \\
\hline 1st 10 States & 7.08 & 0.52 & 7.24 \\
\hline All States & 8.94 & 1.36 & 9.04 \\
\hline \multicolumn{4}{|l|}{${ }^{32} S$} \\
\hline 1st 10 States & 8.81 & 0.41 & 9.45 \\
\hline All States & 10.19 & 1.24 & 10.91 \\
\hline \multicolumn{4}{|l|}{${ }^{36} A r$} \\
\hline 1st 10 States & 5.10 & 0.48 & 5.21 \\
\hline All States & 5.48 & 0.90 & 5.72 \\
\hline \multicolumn{4}{|l|}{$\overline{{ }^{44} \mathrm{Ti}}$} \\
\hline 1st 10 States & 3.73 & 1.32 & 1.26 \\
\hline All States & 5.56 & 2.13 & 3.52 \\
\hline
\end{tabular}


TABLE V. $B_{l}, B_{\sigma}$ and $B(M 1)$ strengths in $\mu_{N}^{2}$ for states with $B(M 1)$ (usually) $\geq 0.3 \mu_{N}^{2}$ as a function of $x$ and $y$-the strengths of the spin-orbit and tensor interactions respectively

\begin{tabular}{|c|c|c|c|c|c|c|}
\hline Nucleus & $x$ & $y$ & $E_{x}(\mathrm{MeV})$ & $B_{l}$ & $B_{\sigma}$ & $\overline{B(M 1)}$ \\
\hline \multirow[t]{4}{*}{${ }^{8} \mathrm{Be}$} & 1 & 1 & 13.72 & 0.257 & 0.115 & 0.716 \\
\hline & 1.5 & 1 & 13.06 & 0.267 & 0.218 & 0.967 \\
\hline & 1 & 0.5 & 13.75 & 0.255 & 0.139 & 0.770 \\
\hline & 1.5 & 0.5 & 13.05 & 0.258 & 0.249 & 1.014 \\
\hline \multirow[t]{5}{*}{${ }^{10} \mathrm{Be}$} & 1 & 1 & 7.68 & 0.017 & 1.512 & 1.849 \\
\hline & 1.5 & 1 & 8.84 & 0.000 & 2.837 & 2.837 \\
\hline & 1 & 0.5 & 7.71 & 0.001 & 2.333 & 1.966 \\
\hline & 1.5 & 0.5 & 8.45 & 0.004 & 0.856 & 0.774 \\
\hline & & & 9.26 & 0.000 & 2.232 & 2.695 \\
\hline \multirow[t]{4}{*}{$\overline{{ }^{12} C}$} & 1 & 1 & 13.60 & 0.031 & 0.583 & 0.886 \\
\hline & 1.5 & 1 & 13.08 & 0.004 & 1.710 & 1.887 \\
\hline & 1 & 0.5 & 13.33 & 0.012 & 0.901 & 1.121 \\
\hline & 1.5 & 0.5 & 13.11 & 0.000 & 2.571 & 2.545 \\
\hline \multirow[t]{6}{*}{$\overline{{ }^{20} \mathrm{Ne}}$} & 1 & 1 & 12.40 & 0.434 & 0.064 & 0.830 \\
\hline & & & 19.21 & 0.136 & 0.047 & 0.344 \\
\hline & 1.5 & 1 & 10.73 & 0.470 & 0.288 & 1.494 \\
\hline & 1 & 0.5 & 12.18 & 0.240 & 0.003 & 0.187 \\
\hline & & & 12.25 & 0.238 & 0.136 & 0.735 \\
\hline & 1.5 & 0.5 & 10.66 & 0.475 & 0.346 & 1.631 \\
\hline \multirow[t]{2}{*}{$\Delta \epsilon_{s}=2 M e V$} & 1.5 & 0.5 & 9.757 & 0.511 & 0.623 & 2.263 \\
\hline & & & 16.91 & 0.037 & 0.532 & 0.307 \\
\hline \multirow[t]{9}{*}{${ }^{22} \mathrm{Ne}$} & 1 & 1 & 8.45 & 0.005 & 1.121 & $\overline{1.464}$ \\
\hline & 1.5 & 1 & 7.06 & 0.176 & 0.053 & 0.461 \\
\hline & & & 8.10 & 0.015 & 0.875 & 0.944 \\
\hline & & & 10.00 & 0.006 & 0.370 & 0.374 \\
\hline & & & 10.61 & 0.012 & 1.086 & 1.359 \\
\hline & 1 & 0.5 & 8.63 & 0.001 & 1.492 & 1.800 \\
\hline & 1.5 & 0.5 & 6.99 & 0.199 & 0.170 & 0.785 \\
\hline & & & 8.57 & 0.011 & 1.077 & 1.065 \\
\hline & & & 10.95 & 0.001 & 1.275 & 1.518 \\
\hline \multirow[t]{3}{*}{$\Delta \epsilon_{s}=2 M e V$} & 1.5 & 0.5 & 7.320 & 0.152 & 0.732 & 1.711 \\
\hline & & & 8.575 & 0.009 & 1.664 & 1.544 \\
\hline & & & 11.54 & 0.004 & 1.111 & 1.279 \\
\hline \multirow[t]{6}{*}{${ }^{24} \mathrm{Mg}$} & 1 & 1 & 10.27 & 0.280 & 0.492 & 1.516 \\
\hline & 1.5 & 1 & 9.35 & 0.204 & 0.943 & 2.024 \\
\hline & & & 12.66 & 0.076 & 0.077 & 0.304 \\
\hline & 1 & 0.5 & 10.17 & 0.280 & 0.595 & 1.693 \\
\hline & 1.5 & 0.5 & 9.32 & 0.195 & 1.024 & 2.111 \\
\hline & & & 9.466 & 0.083 & 0.265 & 0.644 \\
\hline
\end{tabular}




\begin{tabular}{|c|c|c|c|c|c|c|}
\hline \multirow[t]{2}{*}{$\Delta \epsilon_{s}=2 M e V$} & \multirow[t]{2}{*}{1.5} & \multirow[t]{2}{*}{0.5} & \multirow{2}{*}{$\begin{array}{l}9.401 \\
14.89\end{array}$} & \multirow{2}{*}{$\begin{array}{c}0.183 \\
0.06\end{array}$} & \multirow{2}{*}{$\begin{array}{l}2.316 \\
0.163\end{array}$} & \multirow{2}{*}{$\begin{array}{l}3.799 \\
0.422\end{array}$} \\
\hline & & & & & & \\
\hline \multirow[t]{9}{*}{$\overline{{ }^{28} \mathrm{Si}}$} & 1 & 1 & 10.31 & 0.055 & 0.860 & 1.352 \\
\hline & & & 11.78 & 0.122 & 0.123 & 0.491 \\
\hline & & & 12.97 & 0.359 & 0.001 & 0.387 \\
\hline & & & 14.53 & 0.399 & 0.002 & 0.350 \\
\hline & 1.5 & 1 & 9.64 & 0.075 & 2.810 & 3.801 \\
\hline & 1 & 0.5 & 10.11 & 0.064 & 1.413 & 2.06 \\
\hline & & & 14.54 & 0.383 & 0.001 & 0.352 \\
\hline & 1.5 & 0.5 & 9.44 & 0.221 & 2.266 & 3.903 \\
\hline & & & 10.54 & 0.062 & 1.313 & 0.804 \\
\hline \multirow[t]{4}{*}{$\Delta \epsilon_{s}=2 M e V$} & 1.5 & 0.5 & 10.03 & 0.007 & 4.258 & 4.612 \\
\hline & & & 10.99 & 0.005 & 0.486 & 0.395 \\
\hline & & & 11.64 & 0.028 & 1.073 & 0.756 \\
\hline & & & 12.71 & 0.136 & 0.252 & 0.759 \\
\hline \multirow[t]{12}{*}{$\overline{{ }^{32} S}$} & 1 & 1 & 10.28 & 0.009 & 0.855 & 1.039 \\
\hline & & & 11.17 & 0.111 & 0.266 & 0.721 \\
\hline & & & 12.12 & 0.423 & 0.009 & 0.555 \\
\hline & 1.5 & 1 & 9.63 & 0.007 & 4.574 & 4.212 \\
\hline & & & 10.53 & 0.001 & 1.378 & 1.447 \\
\hline & & & 11.77 & 0.163 & 0.242 & 0.801 \\
\hline & 1 & 0.5 & 9.78 & 0.001 & 1.447 & 1.529 \\
\hline & & & 11.14 & 0.097 & 0.753 & 1.391 \\
\hline & 1.5 & 0.5 & 10.05 & 0.017 & 3.372 & 2.912 \\
\hline & & & 11.21 & 0.018 & 5.976 & 5.341 \\
\hline & & & 13.01 & 0.116 & 0.231 & 0.676 \\
\hline & & & 13.81 & 0.105 & 0.979 & 0.442 \\
\hline \multirow[t]{9}{*}{$\overline{{ }^{36} A r}$} & 1 & 1 & 10.70 & 0.002 & 1.180 & 1.290 \\
\hline & & & 13.79 & 0.126 & 0.242 & 0.718 \\
\hline & & & 15.50 & 0.237 & 0.005 & 0.311 \\
\hline & 1.5 & 1 & 11.04 & 0.009 & 2.699 & 2.433 \\
\hline & & & 12.10 & 0.053 & 0.105 & 0.307 \\
\hline & & & 16.66 & 0.000 & 0.488 & 0.491 \\
\hline & 1 & 0.5 & 10.97 & 0.016 & 1.639 & 1.981 \\
\hline & & & 12.29 & 0.194 & 0.057 & 0.460 \\
\hline & & & 16.41 & 0.071 & 0.174 & 0.466 \\
\hline
\end{tabular}




\begin{tabular}{|c|c|c|c|c|c|c|}
\hline & 1.5 & 0.5 & 11.91 & 0.018 & 3.069 & 2.622 \\
\hline & & & 12.97 & 0.041 & 1.010 & 1.457 \\
\hline & & & 13.73 & 0.017 & 0.376 & 0.553 \\
\hline & & & 17.65 & 0.002 & 0.431 & 0.488 \\
\hline \multirow[t]{6}{*}{$\Delta \epsilon_{s}=2 M e V$} & 1.5 & 0.5 & 10.78 & 0.073 & 0.817 & 0.402 \\
\hline & & & 12.02 & 0.028 & 1.900 & 2.386 \\
\hline & & & 12.30 & 0.025 & 0.519 & 0.771 \\
\hline & & & 14.87 & 0.016 & 0.526 & 0.387 \\
\hline & & & 15.16 & 0.027 & 0.509 & 0.300 \\
\hline & & & 16.52 & 0.006 & 0.471 & 0.584 \\
\hline \multirow[t]{8}{*}{$\overline{{ }^{44} T i}$} & 1 & 1 & 9.22 & 0.275 & 0.122 & 0.763 \\
\hline & & & 11.91 & 0.527 & 0.015 & 0.365 \\
\hline & 1.5 & 1 & 9.01 & 0.407 & 0.397 & 1.610 \\
\hline & & & 9.50 & 0.356 & 0.007 & 0.465 \\
\hline & 1 & 0.5 & 9.19 & 0.266 & 0.148 & 0.810 \\
\hline & & & 12.76 & 0.020 & 0.205 & 0.352 \\
\hline & & & 8.98 & 0.471 & 0.474 & 1.889 \\
\hline & & & 9.49 & 0.303 & 0.001 & 0.340 \\
\hline
\end{tabular}


TABLE VI. $B_{l}, B_{\sigma}$ and $B(M 1)$ strengths in $\mu_{N}^{2}$ for states of interest not contained in Table X, and for $x=1.5 y=0.5$

\begin{tabular}{|c|c|c|c|c|}
\hline Nucleus & $E_{x}(M e V)$ & $B_{l}$ & $B_{\sigma}$ & $B(M 1)$ \\
\hline \multirow[t]{2}{*}{$\overline{{ }^{8} \mathrm{Be}}$} & 17.42 & 0.230 & 0.185 & 0.003 \\
\hline & 19.51 & 0.141 & 0.023 & 0.050 \\
\hline${ }^{10} \mathrm{Be}$ & 16.11 & 0.020 & 0.000 & 0.025 \\
\hline \multirow[t]{2}{*}{$T=1$} & 19.50 & 0.026 & 0.001 & 0.011 \\
\hline & 20.57 & 0.039 & 0.010 & 0.009 \\
\hline \multirow[t]{2}{*}{$T=2$} & 18.74 & 0.089 & 0.217 & 0.028 \\
\hline & 23.47 & 0.046 & 0.012 & 0.010 \\
\hline \multirow[t]{2}{*}{${ }^{12} C$} & 17.46 & 0.284 & 0.074 & 0.068 \\
\hline & 20.18 & 0.167 & 0.005 & 0.112 \\
\hline \multirow[t]{2}{*}{${ }^{20} \mathrm{Ne}$} & 11.50 & 0.136 & 0.108 & 0.001 \\
\hline & 18.09 & 0.239 & 0.361 & 0.013 \\
\hline$\Delta \epsilon_{s}=2 M e V$ & 12.80 & 0.290 & 0.162 & 0.018 \\
\hline \multirow[t]{3}{*}{${ }^{24} M g$} & 12.14 & 0.205 & 0.001 & 0.235 \\
\hline & 13.92 & 0.237 & 0.384 & 0.017 \\
\hline & 15.00 & 0.115 & 0.002 & 0.146 \\
\hline$\Delta \epsilon_{s}=2 M e V$ & 13.08 & 0.177 & 0.144 & 0.002 \\
\hline${ }^{28} S i$ & 13.26 & 0.142 & 0.381 & 0.058 \\
\hline \multirow[t]{3}{*}{$\Delta \epsilon_{s}=2 M e V$} & 10.79 & 0.104 & 0.003 & 0.140 \\
\hline & 13.25 & 0.196 & 0.682 & 0.147 \\
\hline & 14.47 & 0.003 & 0.214 & 0.168 \\
\hline${ }^{32} S$ & 17.01 & 0.140 & 0.021 & 0.270 \\
\hline \multirow[t]{2}{*}{$\Delta \epsilon_{s}=2 M e V$} & 10.98 & 0.149 & 0.525 & 0.114 \\
\hline & 15.10 & 0.106 & 0.415 & 0.015 \\
\hline${ }^{36} A r$ & 7.80 & 0.305 & 0.320 & 0.000 \\
\hline$\Delta \epsilon_{s}=2 M e V$ & 7.93 & 0.291 & 0.271 & 0.000 \\
\hline \multirow[t]{2}{*}{${ }^{44} T i$} & 11.52 & 0.127 & 0.028 & 0.035 \\
\hline & 12.77 & 0.095 & 0.469 & 0.142 \\
\hline$\Delta \epsilon_{p}=2 M e V$ & 9.93 & 0.323 & 0.130 & 0.043 \\
\hline
\end{tabular}


TABLE VII. Isoscalar and Isovector $B(E 2)$ (in $e^{2} f m^{4}$ ) as a function of the strengths $(x, y)$ of the spin-orbit and tensor interaction in small space.

\begin{tabular}{|c|c|c|c|c|}
\hline Nucleus & $x$ & $y$ & $B(E 2)_{\text {isoscalar }}^{a}$ & $B(E 2)_{\text {isovector }}^{b}$ \\
\hline \multirow[t]{4}{*}{${ }^{8} \mathrm{Be}$} & 1 & 1 & 71.27 & 9.26 \\
\hline & 1.5 & 1 & 70.41 & 10.19 \\
\hline & 1 & 0.5 & 71.24 & 9.35 \\
\hline & 1.5 & 0.5 & 70.16 & 10.44 \\
\hline${ }^{10} \mathrm{Be}$ & 1 & 1 & 73.18 & 29.83 \\
\hline \multirow[t]{3}{*}{$T=1 \rightarrow T=1$} & 1.5 & 1 & 71.49 & 27.90 \\
\hline & 1 & 0.5 & 72.44 & 28.47 \\
\hline & 1.5 & 0.5 & 70.29 & 27.38 \\
\hline${ }^{10} \mathrm{Be}$ & 1 & 1 & 0 & 3.144 \\
\hline \multirow[t]{3}{*}{$T=1 \rightarrow T=2$} & 1.5 & 1 & 0 & 3.512 \\
\hline & 1 & 0.5 & 0 & 3.164 \\
\hline & 1.5 & 0.5 & 0 & 3.564 \\
\hline \multirow[t]{4}{*}{${ }^{12} C$} & 1 & 1 & 82.73 & 13.56 \\
\hline & 1.5 & 1 & 78.43 & 17.72 \\
\hline & 1 & 0.5 & 82.00 & 14.16 \\
\hline & 1.5 & 0.5 & 75.30 & 19.20 \\
\hline \multirow[t]{4}{*}{${ }^{20} \mathrm{Ne}$} & 1 & 1 & 315.1 & 53.44 \\
\hline & 1.5 & 1 & 313.5 & 45.24 \\
\hline & 1 & 0.5 & 316.0 & 52.68 \\
\hline & 1.5 & 0.5 & 311.1 & 44.56 \\
\hline$\Delta \epsilon_{s}=2 M e V$ & 1.5 & 0.5 & 266.7 & 39.80 \\
\hline${ }^{22} \mathrm{Ne}$ & 1 & 1 & 352.7 & 108.3 \\
\hline \multirow[t]{3}{*}{$T=1 \rightarrow T=1$} & 1.5 & 1 & 350.4 & 101.1 \\
\hline & 1 & 0.5 & 353.8 & 107.9 \\
\hline & 1.5 & 0.5 & 344.8 & 98.64 \\
\hline$\Delta \epsilon_{s}=2 M e V$ & 1.5 & 0.5 & 298.0 & 90.17 \\
\hline${ }^{22} \mathrm{Ne}$ & 1 & 1 & 0 & 22.12 \\
\hline \multirow[t]{3}{*}{$T=1 \rightarrow T=2$} & 1.5 & 1 & 0 & 16.39 \\
\hline & 1 & 0.5 & 0 & 21.26 \\
\hline & 1.5 & 0.5 & 0 & 15.12 \\
\hline$\Delta \epsilon_{s}=2 M e V$ & 1.5 & 0.5 & 0 & 14.48 \\
\hline \multirow[t]{4}{*}{${ }^{24} M g$} & 1 & 1 & 515.8 & 71.07 \\
\hline & 1.5 & 1 & 511.3 & 78.89 \\
\hline & 1 & 0.5 & 515.6 & 72.87 \\
\hline & 1.5 & 0.5 & 508.9 & 80.68 \\
\hline$\Delta \epsilon_{s}=2 \mathrm{MeV}$ & 1.5 & 0.5 & 481.8 & 82.86 \\
\hline${ }^{28} S i^{c}$ & 1 & 1 & 651.8 & $\mathrm{~N} / \mathrm{A}$ \\
\hline
\end{tabular}




\begin{tabular}{|c|c|c|c|c|}
\hline & 1.5 & 1 & 574.0 & $\mathrm{~N} / \mathrm{A}$ \\
\hline & 1 & 0.5 & 635.4 & $\mathrm{~N} / \mathrm{A}$ \\
\hline & 1.5 & 0.5 & 546.9 & 26.4 \\
\hline$\Delta \epsilon_{s}=2 M e V$ & 1.5 & 0.5 & 439.5 & $\mathrm{~N} / \mathrm{A}$ \\
\hline \multirow[t]{4}{*}{${ }^{32} S$} & 1 & 1 & 529.8 & 80.41 \\
\hline & 1.5 & 1 & 337.2 & 123.8 \\
\hline & 1 & 0.5 & 488.5 & 89.66 \\
\hline & 1.5 & 0.5 & 254.8 & 130.3 \\
\hline$\Delta \epsilon_{s}=2 M e V$ & 1.5 & 0.5 & 321.7 & 124.7 \\
\hline \multirow[t]{4}{*}{${ }^{36} \mathrm{Ar}$} & 1 & 1 & 331.2 & 59.01 \\
\hline & 1.5 & 1 & 289.0 & 83.00 \\
\hline & 1 & 0.5 & 322.6 & 63.87 \\
\hline & 1.5 & 0.5 & 280.9 & 85.71 \\
\hline$\Delta \epsilon_{s}=2 M e V$ & 1.5 & 0.5 & 307.9 & 75.91 \\
\hline \multirow[t]{4}{*}{$\overline{{ }^{44} T i}$} & 1 & 1 & 963.9 & 119.8 \\
\hline & 1.5 & 1 & 953.7 & 109.9 \\
\hline & 1 & 0.5 & 967.1 & 120.3 \\
\hline & 1.5 & 0.5 & 946.9 & 109.2 \\
\hline$\Delta \epsilon_{p}=2 M e V$ & 1.5 & 0.5 & & \\
\hline
\end{tabular}

(a) $e_{p}=1.5, e_{n}=0.5$

(b) $e_{p}=1, e_{n}=-1$

(c) For $(x, y)=(1,1)$ the sum is over the 500 lowest states, but for all the other $(x, y)$ pairs the sum is over the lowest 50 states only.

TABLE VIII. B(M1) strength in ${ }^{12} \mathrm{C}$ in $0 \hbar \omega$ space $(0 \hbar \omega)$.

\begin{tabular}{lcccc}
\hline & \multicolumn{2}{c}{$1^{+} ; 0$ state } & \multicolumn{2}{c}{$1^{+} ; 1$ state } \\
& $E_{x}$ & $\mathrm{~B}(\mathrm{M} 1)^{\mathrm{a}}$ & $E_{x}$ & $\mathrm{~B}(\mathrm{M} 1)$ \\
& $(\mathrm{MeV})$ & $\left(10^{-2} \mu_{N}^{2}\right)$ & $(\mathrm{MeV})$ & $\left(\mu_{N}^{2}\right)$ \\
\hline Experiment & 12.71 & $4.0(3)$ & 15.11 & $2.63(8)$ \\
$\mathrm{X}=1, \mathrm{Y}=1$ & 11.80 & 0.26 & 13.60 & 0.89 \\
$\mathrm{X}=1.5, \mathrm{Y}=1$ & 11.03 & 0.86 & 13.08 & 1.89 \\
$\mathrm{X}=1, \mathrm{Y}=0.5$ & 12.30 & 0.54 & 13.33 & 1.12 \\
$\mathrm{X}=1.5, \mathrm{Y}=0.5$ & 11.80 & 1.65 & 13.11 & 2.55 \\
\hline \hline
\end{tabular}

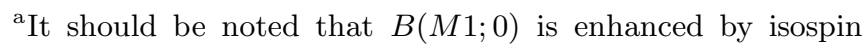
mixing. One should compare the results of our calculations to the Cohen-Kurath shell model calculation which found that $B(M 1 ; 0)=1.4 \times 10^{-2} \mu_{N}^{2}$, a value which agrees well with our results, especially for $x=1.5 y=0.5$ 
TABLE IX. B(E2) strength in ${ }^{12} \mathrm{C}$

\begin{tabular}{|c|c|c|c|c|}
\hline \multirow[b]{2}{*}{$\left(e_{p}, e_{n}\right)$} & \multicolumn{2}{|c|}{$2^{+} ; 0$ state } & \multicolumn{2}{|c|}{$2^{+} ; 1$ state } \\
\hline & $\begin{array}{c}E_{x} \\
(\mathrm{MeV})\end{array}$ & $\begin{array}{c}B(E 2) \\
\mathrm{e}^{2} \mathrm{fm}^{4} \\
(1.5,0.5) \\
\end{array}$ & $\begin{array}{c}E_{x} \\
(\mathrm{MeV})\end{array}$ & $\begin{array}{c}B(E 2) \\
\mathrm{e}^{2} \mathrm{fm}^{4} \\
(1.5,0.5) \\
\end{array}$ \\
\hline Expt & 4.44 & $39.9(2.2)$ & 16.11 & $2.0(0.2)$ \\
\hline $\mathrm{X}=1, \mathrm{Y}=1$ & 3.80 & 80.6 & 15.78 & 2.6 \\
\hline $\mathrm{X}=1.5, \mathrm{Y}=1$ & 3.82 & 75.3 & 14.44 & 3.1 \\
\hline \multirow[t]{2}{*}{$\mathrm{X}=1, \mathrm{Y}=0.5$} & 3.79 & 80.1 & 14.86 & 1.2 \\
\hline & & & 15.58 & 1.6 \\
\hline $\mathrm{X}=1.5, \mathrm{Y}=0.5$ & 4.11 & 72.9 & 14.25 & 3.8 \\
\hline
\end{tabular}

TABLE X. B(M1) strength in ${ }^{20} \mathrm{Ne}$.

\begin{tabular}{lcccc}
\hline \hline \multicolumn{5}{c}{$1^{+} ; 1$ state } \\
& $E_{x}$ & $B(M 1)^{\mathrm{a}}$ & $B_{\sigma}$ & $B_{l}$ \\
& $(\mathrm{MeV})$ & $\left(\mu_{N}^{2}\right)$ & $\left(\mu_{N}^{2}\right)$ & $\left(\mu_{N}^{2}\right)$ \\
\hline Expt & 11.26 & $2.02(36)$ & $0.49(6)$ & $0.52(15)$ \\
$\mathrm{X}=1, \mathrm{Y}=1$ & 12.40 & 0.83 & 0.06 & 0.43 \\
$\mathrm{X}=1.5, \mathrm{Y}=1$ & 10.73 & 1.49 & 0.29 & 0.47 \\
$\mathrm{X}=1, \mathrm{Y}=0.5$ & 12.19 & 0.19 & 0.03 & 0.24 \\
& 12.25 & 0.73 & 0.14 & 0.24 \\
$\mathrm{X}=1.5, \mathrm{Y}=0.5$ & 10.61 & 1.63 & 0.35 & 0.47 \\
\hline \hline
\end{tabular}

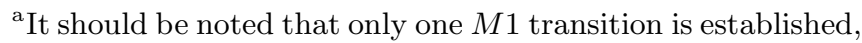
but $B_{\sigma}$ and $B_{l}$ can be separated from comparison of (e, $\left.\mathrm{e}^{\prime}\right)$ and $\left(\mathrm{p}, \mathrm{p}^{\prime}\right)$. Constructive interference assumed. 
TABLE XI. B(E2) strength in ${ }^{20} \mathrm{Ne}$

\begin{tabular}{lcc|cc}
\hline \hline & \multicolumn{2}{c}{} & & \\
& \multicolumn{2}{c}{$2^{+} ; 0$ state } & \multicolumn{2}{c}{$2^{+} ; 0$ state } \\
& $E_{x}$ & $B(E 2)$ & $E_{x}$ & $B(E 2)$ \\
& $(\mathrm{MeV})$ & $\left(\mathrm{e}^{2} \mathrm{fm}^{4}\right)$ & $(\mathrm{MeV})$ & $\left(\mathrm{e}^{2} \mathrm{fm}^{4}\right)$ \\
$\left(e_{p}, e_{n}\right)$ & & $(1.5,0.5)$ & & $(1.5,0.5)$ \\
\hline Expt & 1.63 & $340(22)$ & 7.42 & $<0.83$ \\
& 7.83 & $12.0(1.5)$ & 10.27 & $1.4(4)$ \\
\hline $\mathrm{X}=1, \mathrm{Y}=1$ & 2.92 & 299 & 9.21 & 2.9 \\
& 11.82 & 4.4 & 11.12 & 4.1 \\
& & & 11.76 & 0.97 \\
\hline $\mathrm{X}=1.5, \mathrm{Y}=1$ & 2.40 & 298 & 7.82 & 0.95 \\
& 10.14 & 0.7 & & \\
& 10.75 & 3.6 & 9.90 & 1.3 \\
& 11.08 & 0.4 & 10.76 & 3.0 \\
\hline $\mathrm{X}=1, \mathrm{Y}=0.5$ & 2.84 & 301 & 9.01 & 2.6 \\
& 11.08 & 0.4 & 11.20 & 3.9 \\
& 11.74 & 3.8 & 11.65 & 1.1 \\
\hline $\mathrm{X}=1.5, \mathrm{Y}=0.5$ & 2.37 & 296 & 7.61 & 0.73 \\
& 10.05 & 1.8 & 9.81 & 1.7 \\
& 10.73 & 3.0 & 10.90 & 2.5 \\
\hline \hline
\end{tabular}

TABLE XII. B(M1) strength in ${ }^{22} \mathrm{Ne}$

\begin{tabular}{|c|c|c|c|c|c|c|}
\hline & \multicolumn{2}{|c|}{$1^{+} ; 1$ state } & \multicolumn{2}{|c|}{$1^{+} ; 1$ state } & \multicolumn{2}{|c|}{$1^{+} ; 1$ state } \\
\hline & $\begin{array}{c}E_{x} \\
(\mathrm{MeV})\end{array}$ & $\begin{array}{c}B(M 1) \\
\left(\mu_{N}^{2}\right)\end{array}$ & $\begin{array}{c}E_{x} \\
(\mathrm{MeV})\end{array}$ & $\begin{array}{c}B(M 1) \\
\left(\mu_{N}^{2}\right)\end{array}$ & $\begin{array}{c}E_{x} \\
(\mathrm{MeV})\end{array}$ & $\begin{array}{c}B(M 1) \\
\left(\mu_{N}^{2}\right)\end{array}$ \\
\hline $\begin{array}{l}\text { Expt. } \\
(x, y)\end{array}$ & 5.33 & $0.43(17)$ & 6.85 & $1.36(56)$ & 9.18 & $\overline{1.80(7)}$ \\
\hline$(1,1)$ & 5.70 & 0.02 & 6.85 & 0.14 & 8.45 & 1.46 \\
\hline$(1.5,1)$ & 7.06 & 0.46 & 8.10 & 0.94 & 10.61 & 1.36 \\
\hline$(1,0.5)$ & 5.59 & 0.007 & 7.76 & 0.15 & 8.63 & 1.80 \\
\hline$(1.5,0.5)$ & 6.99 & 0.79 & 8.56 & 1.07 & 10.95 & 1.52 \\
\hline
\end{tabular}


TABLE XIII. B(E2) strength in ${ }^{22} \mathrm{Ne}$.

\begin{tabular}{lcc|cc}
\hline \hline & \multicolumn{2}{c}{} & \multicolumn{2}{c}{$2^{+} ; 1$ state } \\
\hline & $2_{x} 2^{+} ;$state & $B(E 2)$ & $E_{x}$ & $B(E 2)$ \\
& $(\mathrm{MeV})$ & $\left(\mathrm{e}^{2} \mathrm{fm}^{4}\right)$ & $(\mathrm{MeV})$ & $\left(\mathrm{e}^{2} \mathrm{fm}^{4}\right)$ \\
$\left(e_{p}, e_{n}\right)^{\mathrm{a}}$ & $(1.5,0.5)$ & $(1.5,0.5)$ & & \\
\hline Expt & 1.27 & $235(3)$ & 4.46 & $>4.6$ \\
& 6.12 & $3.5(1.5)$ & 7.64 & $19(8)$ \\
\hline $\mathrm{X}=1, \mathrm{Y}=1$ & 2.27 & 199 & 3.10 & 113 \\
& 4.21 & 2.9 & 5.85 & 12.4 \\
\hline $\mathrm{X}=1.5, \mathrm{Y}=1$ & 1.98 & 289 & 2.77 & 7.5 \\
& 4.27 & 8.7 & 5.03 & 22.9 \\
\hline $\mathrm{X}=1, \mathrm{Y}=0.5$ & 2.28 & 243 & 3.02 & 67 \\
& 4.18 & 6.5 & 5.72 & 13.0 \\
\hline $\mathrm{X}=1.5, \mathrm{Y}=0.5$ & 1.90 & 290 & 3.00 & 0.004 \\
& 4.43 & 7.1 & 4.88 & 26.1 \\
\hline \hline
\end{tabular}

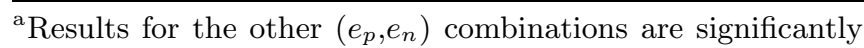
worse. It should be noted that there is no experimental evidence for isovector $E 2$ transitions. 
TABLE XIV. Experimental $B(M 1)$ values ${ }^{a}$ for ${ }^{24} M g$

\begin{tabular}{|c|c|}
\hline $\begin{array}{l}E_{x} \\
\mathrm{MeV} \\
\end{array}$ & $\begin{array}{c}B(M 1) \uparrow \\
\left(\mu_{N}^{2}\right)\end{array}$ \\
\hline 8.865 & $0.042 \pm 0.011$ \\
\hline 9.820 & $0.260 \pm 0.040$ \\
\hline 9.962 & $1.160 \pm 0.150$ \\
\hline 10.711 & $3.180 \pm 0.300$ \\
\hline 10.918 & $0.100 \pm 0.020$ \\
\hline 11.002 & $0.048 \pm 0.011$ \\
\hline 11.373 & $0.056 \pm 0.013$ \\
\hline 11851 & $0.033 \pm 0.008$ \\
\hline 11.960 & $0.046 \pm 0.010$ \\
\hline 12.145 & $0.035 \pm 0.012$ \\
\hline 12.264 & $0.035 \pm 0.009$ \\
\hline 12.406 & $0.047 \pm 0.010$ \\
\hline 12.527 & $0.065 \pm 0.011$ \\
\hline 12.807 & $0.179 \pm 0.018$ \\
\hline 12.961 & $0.151 \pm 0.012$ \\
\hline 13.081 & $0.024 \pm 0.011$ \\
\hline 13.222 & $0.067 \pm 0.009$ \\
\hline 13.675 & $0.030 \pm 0.008$ \\
\hline 13.940 & $0.201 \pm 0.014$ \\
\hline 14.094 & $0.026 \pm 0.008$ \\
\hline 14.267 & $0.059 \pm 0.009$ \\
\hline
\end{tabular}

(a) obtained with the use of the Wildenthal-Brown form factor.

TABLE XV. Experimental Excitation Energies and $B_{\sigma}$ values $^{a}$ for $1^{+} T=1$ states ${ }^{24} M g$

\begin{tabular}{lc}
\hline \hline$E_{x}$ & $B_{\sigma}$ \\
$\mathrm{MeV}$ & $\left(\mu_{N}^{2}\right)$ \\
\hline $9.83(T=0 ?)$ & $0.29 \pm 0.02$ \\
9.97 & $0.38 \pm 0.03$ \\
10.72 & $2.75 \pm 0.20$ \\
12.53 & $0.60 \pm 0.03$ \\
12.82 & $0.85 \pm 0.03$ \\
12.96 & $0.37 \pm 0.02$ \\
13.90 & $0.56 \pm 0.09$ \\
16.12 & $0.31 \pm 0.06$ \\
\hline \hline
\end{tabular}

(a) Taken from $\left(p, p^{\prime}\right)$ data by Crawley et.al. [18]. 
TABLE XVI. Calculated $B(M 1) \quad \uparrow$ strengths to $J=1^{+} T=1$ states in ${ }^{24} M g$ obtained with the Realistic Interaction $(x=1.5 y=0.5)$.

\begin{tabular}{lccc}
\hline \hline$E_{x}$ & $B(M 1)_{\text {physical }}$ & $\begin{array}{c}B_{l} \\
\left(\mu_{N}^{2}\right)\end{array}$ & $\begin{array}{c}B_{\sigma} \\
\left(\mu_{N}^{2}\right)\end{array}$ \\
\hline 9.33 & $\left(\mu_{N}^{2}\right)$ & 0.19 & 1.02 \\
9.47 & 2.11 & 0.08 & 0.27 \\
10.50 & 0.64 & 0.00 & 0.03 \\
11.91 & 0.03 & 0.07 & 0.03 \\
12.14 & 0.19 & 0.21 & 0.001 \\
13.49 & 0.23 & 0.01 & 0.008 \\
13.92 & 0.03 & 0.24 & 0.38 \\
14.24 & 0.02 & 0.02 & 0.00 \\
14.59 & 0.02 & 0.00 & 0.05 \\
15.00 & 0.03 & 0.12 & 0.002 \\
\hline Sum $^{2}(10)^{a}$ & 0.15 & 0.94 & 1.79 \\
Total Sum $^{b}$ & 3.45 & 1.69 & 4.04 \\
\hline \hline
\end{tabular}

(a) Sum to the lowest 10 states shown in the table.

(b) Sum to all $J=1^{+} T=1$ states in the model space.

TABLE XVII. Calculated $B(M 1) \uparrow$ strengths to $J=1^{+} T=1$ states in ${ }^{24} M g$ obtained with $(x=1.5 y=0.5)$ and $\Delta \epsilon_{s}=2 \mathrm{MeV}$.

\begin{tabular}{lccc}
\hline \hline$E_{x}$ & $B(M 1)_{\text {physical }}$ & $\begin{array}{c}B_{l} \\
\left(\mu_{N}^{2}\right)\end{array}$ & $\begin{array}{c}B_{\sigma} \\
\left(\mu_{N}^{2}\right)\end{array}$ \\
\hline 9.40 & $\left(\mu_{N}^{2}\right)$ & 0.18 & 2.32 \\
9.88 & 3.80 & 0.05 & 0.10 \\
11.08 & 0.00 & 0.04 & 0.01 \\
12.08 & 0.08 & 0.09 & 0.14 \\
13.08 & 0.00 & 0.18 & 0.14 \\
13.33 & 0.00 & 0.06 & 0.00 \\
13.98 & 0.06 & 0.06 & 0.13 \\
14.06 & 0.01 & 0.07 & 0.35 \\
14.89 & 0.10 & 0.06 & 0.16 \\
15.19 & 0.42 & 0.02 & 0.03 \\
\hline Sum(10) & 0.10 & 0.81 & 3.38 \\
Total Sum & 4.58 & 1.63 & 5.46 \\
\hline \hline
\end{tabular}


TABLE XVIII. B(E2) strength in ${ }^{24} \mathrm{Mg}$

\begin{tabular}{|c|c|c|c|c|}
\hline & \multicolumn{2}{|c|}{$2^{+} ; 0$ state } & \multicolumn{2}{|c|}{$2^{+} ; 0$ state } \\
\hline & $E_{x}$ & $B(E 2)$ & $E_{x}$ & $B(E 2)$ \\
\hline & $(\mathrm{MeV})$ & $\left(\mathrm{e}^{2} \mathrm{fm}^{4}\right)$ & $(\mathrm{MeV})$ & $\left(\mathrm{e}^{2} \mathrm{fm}^{4}\right)$ \\
\hline$\left(e_{p}, e_{n}\right)$ & & $(1.5,0.5)$ & & $(1.5,0.5)$ \\
\hline \multirow[t]{2}{*}{ Expt } & 1.369 & $435(9)$ & 4.238 & $33.3(2.4)$ \\
\hline & 7.349 & $11.8(4.7)$ & 9.004 & $3.3(0.6)$ \\
\hline \multirow[t]{2}{*}{$\mathrm{X}=1, \mathrm{Y}=1$} & 2.78 & 488 & 4.33 & 11.9 \\
\hline & 9.32 & 0.6 & 9.93 & 3.8 \\
\hline \multirow[t]{2}{*}{$\mathrm{X}=1.5, \mathrm{Y}=1$} & 2.39 & 474 & 3.74 & 12.8 \\
\hline & 7.87 & 0.8 & 9.67 & 3.5 \\
\hline \multirow[t]{2}{*}{$\mathrm{X}=1, \mathrm{Y}=0.5$} & 2.71 & 486 & 4.24 & 13.4 \\
\hline & 9.07 & 0.8 & 10.0 & 2.7 \\
\hline \multirow[t]{2}{*}{$\mathrm{X}=1.5, \mathrm{Y}=0.5$} & 2.33 & 479 & 3.62 & 4.9 \\
\hline & 7.44 & 1.51 & 9.31 & 4.4 \\
\hline
\end{tabular}

TABLE XIX. Experimental $B(M 1)$ values ${ }^{a}$ for ${ }^{28} S i$

\begin{tabular}{lc}
\hline \hline$E_{x}$ & $B(M 1) \uparrow$ \\
$M e V$ & $\left(\mu_{N}^{2}\right)$ \\
\hline 10.594 & $0.19 \pm 0.04$ \\
10.725 & $0.11 \pm 0.01$ \\
10.901 & $0.90 \pm 0.02$ \\
11.445 & $4.42 \pm 0.20$ \\
12.331 & $0.87 \pm 0.06$ \\
14.030 & $0.37 \pm 0.02$ \\
15.147 & $0.23 \pm 0.02$ \\
15.500 & $0.26 \pm 0.03$ \\
17.56 & $0.18 \pm 0.03$ \\
\hline \hline
\end{tabular}

(a) Obtained through DWBA analysis. 
TABLE XX. Experimental Excitation Energies and $B_{\sigma}$ values ${ }^{a}$ for $1^{+} T=1$ states ${ }^{28} S i$

\begin{tabular}{lc}
\hline \hline$E_{x}$ & $B_{\sigma}$ \\
$\mathrm{MeV}$ & $\left(\mu_{N}^{2}\right)$ \\
\hline 9.72 & $0.39 \pm 0.06$ \\
10.59 & $0.83 \pm 0.83$ \\
10.73 & $0.32 \pm 0.04$ \\
10.82 & $0.21 \pm 0.04$ \\
10.90 & $0.35 \pm 0.05$ \\
11.16 & $0.31 \pm 0.07$ \\
11.45 & $3.32 \pm 0.24$ \\
12.33 & $0.73 \pm 0.14$ \\
12.99 & $0.23 \pm 0.05$ \\
13.35 & $0.81 \pm 0.14$ \\
14.03 & $1.31 \pm 0.12$ \\
15.15 & $0.42 \pm 0.04$ \\
15.50 & $0.12 \pm 0.08$ \\
15.80 & $0.22 \pm 0.02$ \\
Sum & 9.51 \\
\hline \hline
\end{tabular}

(a) Taken from $\left(p, p^{\prime}\right)$ data by Crawley et.al. [18].

TABLE XXI. Calculated $B(M 1) \uparrow$ strengths to $J=1^{+} T=1$ states in ${ }^{28} \mathrm{Si}$ obtained with the Realistic Interaction $(x=1.5 y=0.5)$.

\begin{tabular}{lccc}
\hline \hline$E_{x}$ & $B(M 1)_{\text {physical }}$ & $B_{l}$ & $\begin{array}{c}B_{\sigma} \\
M e V\end{array}$ \\
$\left(\mu_{N}^{2}\right)$ & $\left(\mu_{N}^{2}\right)$ & $\left(\mu_{N}^{2}\right)$ \\
\hline 9.43 & 3.90 & 0.22 & 2.27 \\
10.54 & 0.80 & 0.06 & 1.31 \\
11.05 & 0.00 & 0.03 & 0.02 \\
12.07 & 0.02 & 0.03 & 0.00 \\
12.65 & 0.20 & 0.03 & 0.41 \\
13.27 & 0.06 & 0.14 & 0.38 \\
13.32 & 1.39 & 0.06 & 0.02 \\
14.11 & 0.02 & 0.00 & 0.02 \\
14.61 & 0.11 & 0.06 & 0.33 \\
14.78 & 0.05 & 0.02 & 0.01 \\
\hline Sum $^{(10)^{a}}$ & 5.30 & 0.66 & 4.77 \\
Total Sum & $b$ & 1.46 & 6.97 \\
\hline \hline (a) Sum to the & 7.54 &
\end{tabular}

(a) Sum to the lowest 10 states shown in the table.

(b) Sum to all $J=1^{+} T=1$ states in the model space. 
TABLE XXII. Calculated $B(M 1) \uparrow$ strengths to

$J=1^{+} T=1$ states in ${ }^{28} S i$ obtained with $(x=1.5 y=0.5)$

and $\Delta \epsilon_{s}=2 \mathrm{MeV}$.

\begin{tabular}{lccc}
\hline \hline$E_{x}$ & $B(M 1)_{\text {physical }}$ & $\begin{array}{c}B_{l} \\
\left(\mu_{N}^{2}\right)\end{array}$ & $\begin{array}{c}B_{\sigma} \\
\left(\mu_{N}^{2}\right)\end{array}$ \\
\hline 10.03 & $\left(\mu_{N}^{2}\right)$ & 0.01 & 4.26 \\
10.79 & 4.61 & 0.10 & 0.00 \\
10.99 & 0.14 & 0.00 & 0.49 \\
11.64 & 0.39 & 0.03 & 1.07 \\
12.71 & 0.76 & 0.14 & 0.25 \\
12.82 & 0.76 & 0.00 & 0.01 \\
13.25 & 0.01 & 0.20 & 0.68 \\
13.64 & 0.15 & 0.00 & 0.01 \\
14.08 & 0.00 & 0.04 & 0.25 \\
14.47 & 0.08 & 0.00 & 0.21 \\
\hline Sum(10) & 0.17 & 0.52 & 7.24 \\
Total Sum & 7.08 & 1.36 & 9.04 \\
\hline \hline
\end{tabular}

TABLE XXIII. Experimental $B(E 2)\left(0_{1}^{+} \rightarrow 2{ }_{1}^{+} T\right)$ in ${ }^{28} \mathrm{Si}$

\begin{tabular}{lcc}
\hline \hline$E_{x}$ & $B(E 2)$ & $T$ \\
$(\mathrm{MeV})$ & $\left(e^{2} \mathrm{fm}^{4}\right)$ & \\
1.779 & $334(6)$ & 0 \\
7.381 & $8.5(0.6)$ & 0 \\
7.416 & $4.5(0.5)$ & 0 \\
7.993 & $7.7(1.1)$ & 0 \\
8.259 & $0.73(0.17)$ & 0 \\
9.381 & $1.3(0.5)$ & 1 \\
\hline \hline
\end{tabular}

TABLE XXIV. Theoretical $B(E 2)\left(0_{1}^{+} \rightarrow 2_{1}^{+} T\right)$ in ${ }^{28} \mathrm{Si}$ calculated with $x=1.5 y=0.5\left(e_{p}=1.5 e_{n}=0.5\right)$

\begin{tabular}{lcc}
\hline \hline$E_{x}$ & $B(E 2)$ & $T$ \\
$(\mathrm{MeV})$ & $\left(e^{2} f m^{4}\right)$ & \\
2.41 & 496.7 & 0 \\
4.64 & 4.3 & 0 \\
6.87 & 1.4 & 0 \\
8.00 & 26.7 & 0 \\
8.59 & 8.8 & 1 \\
9.34 & 0.3 & 0 \\
9.92 & 0.7 & 0 \\
\hline \hline
\end{tabular}


TABLE XXV. Experimental Excitation Energies and $B_{\sigma}$ and $B(M 1)$ values ${ }^{a}$ for $1^{+} T=1$ states ${ }^{32} S$

\begin{tabular}{lcc}
\hline \hline$E_{x}$ & $\begin{array}{c}B_{\sigma} \\
\left(\mu_{N}^{2}\right)\end{array}$ & $\begin{array}{c}B(M 1)^{b} \\
\left(\mu_{N}^{2}\right)\end{array}$ \\
\hline 7.63 & $0.42 \pm 0.07$ & \\
7.92 & $0.10 \pm 0.02$ & $1.14 \pm 0.18$ \\
8.13 & $1.46 \pm 0.19$ & $0.69 \pm 0.18$ \\
9.66 & $0.13 \pm 0.02$ & $2.40 \pm 0.22$ \\
11.13 & $4.08 \pm 0.53$ & $1.26 \pm 0.20$ \\
11.63 & $2.38 \pm 0.35$ & \\
11.88 & $0.37 \pm 0.06$ & \\
12.56 & $0.33 \pm 0.06$ & \\
13.90 & $0.24 \pm 0.03$ & \\
14.88 & $0.20 \pm 0.04$ & \\
15.58 & $0.28 \pm 0.05$ & \\
15.70 & $0.16 \pm 0.04$ & \\
15.84 & $0.26 \pm 0.06$ & \\
\hline \hline
\end{tabular}

(a) Taken from $\left(p, p^{\prime}\right)$ data by Crawley et.al. [18].

(b) From $\left(e, e^{\prime}\right)$ data cited by Crawley et.al. 18].

TABLE XXVI. Experimental Excitation Energies and $B(M 1)$ values $^{a}$ for $1^{+} T=1$ states ${ }^{32} S$

\begin{tabular}{lc}
\hline \hline$E_{x}$ & $B(M 1)$ \\
$M e V$ & $\left(\mu_{N}^{2}\right)$ \\
\hline 9.66 & $0.55 \pm 0.24$ \\
9.98 & $0.09 \pm 0.04$ \\
10.45 & $0.10 \pm 0.08$ \\
$10.90^{b}$ & $0.33 \pm 0.04$ \\
11.16 & $1.24 \pm 0.13$ \\
11.50 & $0.10 \pm 0.04$ \\
11.65 & $0.77 \pm 0.14$ \\
12.19 & $0.14 \pm 0.09$ \\
12.65 & $0.11 \pm 0.04$ \\
12.98 & $0.07 \pm 0.04$ \\
$13.41^{b}$ & $0.54 \pm 0.06$ \\
13.78 & $0.39 \pm 0.05$ \\
$13.97^{b}$ & $0.20 \pm 0.11$ \\
14.45 & $0.18 \pm 0.05$ \\
\hline \hline
\end{tabular}

(a) Taken from paper by Petraitis et. al. [20].

(b) May be $M 1$ or $M 2$. 
TABLE XXVII. Calculated $B(M 1) \uparrow$ strengths to $J=1^{+} T=1$ states in ${ }^{32} S$ obtained with the Realistic Interaction $(x=1.5 y=0.5)$.

\begin{tabular}{lccc}
\hline \hline$E_{x}$ & $B(M 1)_{\text {physical }}$ & $B_{l}$ & $B_{\sigma}$ \\
$\mathrm{MeV}$ & $\left(\mu_{N}^{2}\right)$ & $\left(\mu_{N}^{2}\right)$ & $\left(\mu_{N}^{2}\right)$ \\
\hline 10.05 & 2.91 & 0.02 & 3.37 \\
11.21 & 5.34 & 0.02 & 5.98 \\
13.02 & 0.68 & 0.12 & 0.23 \\
13.85 & 0.44 & 0.11 & 0.98 \\
15.10 & 0.04 & 0.06 & 0.22 \\
15.62 & 0.13 & 0.02 & 0.05 \\
15.93 & 0.01 & 0.02 & 0.01 \\
16.55 & 0.16 & 0.02 & 0.07 \\
17.01 & 0.27 & 0.14 & 0.02 \\
17.31 & 0.14 & 0.01 & 0.23 \\
\hline Sum(10) & 10.13 & 0.53 & 11.16 \\
Total Sum & 10.98 & 11.52 & 12.03 \\
\hline \hline
\end{tabular}

TABLE XXVIII. Calculated $B(M 1) \uparrow$ strengths to $J=1^{+} T=1$ states in ${ }^{32} S$ obtained with $(x=1.5 y=0.5)$ and $\Delta \epsilon_{s}=2 \mathrm{MeV}$.

\begin{tabular}{lccc}
\hline \hline$E_{x}$ & $\begin{array}{c}B(M 1)_{\text {physical }} \\
\left(\mu_{N}^{2}\right)\end{array}$ & $\begin{array}{c}B_{l} \\
\left(\mu_{N}^{2}\right)\end{array}$ & $\begin{array}{c}B_{\sigma} \\
\left(\mu_{N}^{2}\right)\end{array}$ \\
\hline 8.58 & 0.40 & 0.01 & 0.53 \\
10.25 & 4.33 & 0.00 & 4.20 \\
10.98 & 0.11 & 0.15 & 0.52 \\
12.08 & 2.64 & 0.01 & 3.02 \\
12.40 & 0.05 & 0.01 & 0.01 \\
13.04 & 0.18 & 0.00 & 0.15 \\
14.22 & 0.02 & 0.06 & 0.15 \\
14.83 & 0.12 & 0.01 & 0.22 \\
15.10 & 0.02 & 0.11 & 0.04 \\
15.17 & 0.95 & 0.04 & 0.59 \\
\hline Sum(10) & 8.81 & 0.41 & 9.45 \\
Total Sum & 10.19 & 1.24 & 10.91 \\
\hline \hline
\end{tabular}


TABLE XXIX. B(E2) strength in ${ }^{32} \mathrm{~S}$

\begin{tabular}{|c|c|c|c|c|}
\hline \multirow[b]{3}{*}{$\left(e_{p}, e_{n}\right)$} & \multicolumn{2}{|c|}{$2^{+} ; 0$ state } & \multicolumn{2}{|c|}{$2^{+} ; 0$ state } \\
\hline & $\begin{array}{c}E_{x} \\
(\mathrm{MeV})\end{array}$ & $\begin{array}{c}B(E 2) \\
\left(\mathrm{e}^{2} \mathrm{fm}^{4}\right)\end{array}$ & $\begin{array}{c}E_{x} \\
(\mathrm{MeV})\end{array}$ & $\begin{array}{c}B(E 2) \\
\left(\mathrm{e}^{2} \mathrm{fm}^{4}\right)\end{array}$ \\
\hline & & $(1.5,0.5)$ & & $(1.5,0.5)$ \\
\hline \multirow[t]{2}{*}{ Expt } & 2.23 & $306(9)$ & 4.49 & $58.1(6.9)$ \\
\hline & 5.55 & $3.9(1.0)$ & 7.12 & ¿. 1.0 \\
\hline \multirow[t]{2}{*}{$\mathrm{X}=1, \mathrm{Y}=1$} & 3.00 & 505 & 4.63 & 10.1 \\
\hline & 9.9 & 0.8 & 11.3 & 1.6 \\
\hline \multirow[t]{2}{*}{$\mathrm{X}=1.5, \mathrm{Y}=1$} & 4.45 & 28.3 & 5.39 & 41.1 \\
\hline & 9.4 & 0.6 & 10.4 & 12.2 \\
\hline \multirow[t]{2}{*}{$\mathrm{X}=1, \mathrm{Y}=0.5$} & 3.19 & 460 & 4.62 & 16.2 \\
\hline & 9.5 & 0.7 & 10.7 & 2.8 \\
\hline \multirow[t]{2}{*}{$X=1.5, Y=0.5$} & 5.77 & 186 & 6.32 & 58.1 \\
\hline & 12.6 & 3.9 & 11.3 & 27.3 \\
\hline
\end{tabular}

TABLE XXX. B(M1) strength in ${ }^{36} \mathrm{Ar}$. Only summed strength is discussed because of the large error bars of the (e, $\left.\mathrm{e}^{\prime}\right)$ experiment.

\begin{tabular}{lc}
\hline \hline & \\
\hline & $\mathrm{B}(\mathrm{M} 1)$ \\
& $\left(\mu_{N}^{2}\right)$ \\
\hline Expt & $2.65 \pm 0.1$ \\
$\mathrm{X}=1, \mathrm{Y}=1$ & 2.30 \\
$\mathrm{X}=1.5, \mathrm{Y}=1$ & 4.09 \\
$\mathrm{X}=1, \mathrm{Y}=0.5$ & 2.68 \\
$\mathrm{X}=1.5, \mathrm{Y}=0.5$ & 4.63 \\
\hline \hline
\end{tabular}


TABLE XXXI. B(E2) strength in ${ }^{36} \mathrm{Ar}$

\begin{tabular}{|c|c|c|c|c|}
\hline \multirow[b]{3}{*}{$\left(e_{p}, e_{n}\right)$} & \multicolumn{2}{|c|}{$2^{+} ; 0$ state } & \multicolumn{2}{|c|}{$2^{+} ; 0$ state } \\
\hline & $\begin{array}{c}E_{x} \\
(\mathrm{MeV})\end{array}$ & $\begin{array}{c}B(E 2) \\
\left(\mathrm{e}^{2} \mathrm{fm}^{4}\right)\end{array}$ & $\begin{array}{c}E_{x} \\
(\mathrm{MeV})\end{array}$ & $\begin{array}{c}B(E 2) \\
\left(\mathrm{e}^{2} \mathrm{fm}^{4}\right)\end{array}$ \\
\hline & & $(1.5,0.5)$ & & $(1.5,0.5)$ \\
\hline Expt & 1.97 & $340(40)$ & 4.44 & $14.0(2.5)$ \\
\hline $\mathrm{X}=1, \mathrm{Y}=1$ & 2.85 & 312 & 7.10 & 2.7 \\
\hline $\mathrm{X}=1.5, \mathrm{Y}=1$ & 2.23 & 247 & 5.96 & 6.3 \\
\hline $\mathrm{X}=1, \mathrm{Y}=0.5$ & 2.77 & 301 & 6.96 & 5.0 \\
\hline $\mathrm{X}=1.5, \mathrm{Y}=0.5$ & 2.19 & 236 & 5.88 & 3.6 \\
\hline
\end{tabular}

TABLE XXXII. B(E2) strength in ${ }^{36} \mathrm{Ar}$ continued

\begin{tabular}{|c|c|c|c|c|}
\hline \multirow[b]{3}{*}{$\left(e_{p}, e_{n}\right)$} & \multicolumn{2}{|c|}{$2^{+} ; 0$ state } & \multicolumn{2}{|c|}{$2^{+} ; 1$ state } \\
\hline & $\begin{array}{c}E_{x} \\
(\mathrm{MeV})\end{array}$ & $\begin{array}{c}B(E 2) \\
\left(\mathrm{e}^{2} \mathrm{fm}^{4}\right)\end{array}$ & $\begin{array}{c}E_{x} \\
(\mathrm{MeV})\end{array}$ & $\begin{array}{c}B(E 2) \\
\left(\mathrm{e}^{2} \mathrm{fm}^{4}\right)\end{array}$ \\
\hline & & $(1.5,0.5)$ & & $(1.5,0.5)$ \\
\hline Expt & 4.95 & $<21.4$ & 6.61 & $2.4(0.9)$ \\
\hline $\mathrm{X}=1, \mathrm{Y}=1$ & 9.39 & 2.2 & 8.56 & 3.6 \\
\hline $\mathrm{X}=1.5, \mathrm{Y}=1$ & 8.19 & 20.8 & 6.34 & 8.0 \\
\hline $\mathrm{X}=1, \mathrm{Y}=0.5$ & 8.81 & 5.4 & 8.19 & 4.6 \\
\hline $\mathrm{X}=1.5, \mathrm{Y}=0.5$ & 8.51 & 30.0 & 6.28 & 8.5 \\
\hline
\end{tabular}

TABLE XXXIII. $B(E 2)$ strength in ${ }^{44} \mathrm{Ti}$

\begin{tabular}{|c|c|c|c|c|}
\hline & \multicolumn{2}{|c|}{$2^{+} ; 0$ state } & \multicolumn{2}{|c|}{$2^{+} ; 0$ state } \\
\hline & $\begin{array}{c}E_{x} \\
(\mathrm{MeV})\end{array}$ & $\begin{array}{c}B(E 2) \\
\left(\mathrm{e}^{2} \mathrm{fm}^{4}\right)\end{array}$ & $\begin{array}{c}E_{x} \\
(\mathrm{MeV})\end{array}$ & $\begin{array}{c}B(E 2) \\
\left(\mathrm{e}^{2} \mathrm{fm}^{4}\right)\end{array}$ \\
\hline Expt & 1.08 & $540(140)$ & 2.53 & $14.2(2.4)$ \\
\hline $\mathrm{X}=1, \mathrm{Y}=1^{a}$ & 2.08 & 924 & 6.12 & 5.0 \\
\hline $\mathrm{X}=1.5, \mathrm{Y}=1$ & 1.67 & 913 & 5.65 & 2.1 \\
\hline $\mathrm{X}=1, \mathrm{Y}=0.5$ & 2.02 & 929 & 6.06 & 4.0 \\
\hline $\mathrm{X}=1.5, \mathrm{Y}=0.5$ & 1.65 & 908 & 5.68 & 2.3 \\
\hline
\end{tabular}

(a) The calculations were done with $e_{p}=1.5, e_{n}=0.5$. 